\title{
A Review for Optical Sensors Based on Photonic Crystal
}

\author{
Cavities \\ Ya-nan Zhang ${ }^{1}$, Yong Zhao ${ }^{1,2, *}$, Ri-qing $\mathrm{Lv}^{1}$ \\ ${ }^{1}$ College of Information Science and Engineering, Northeastern University, Shenyang 110819, \\ China \\ ${ }^{2}$ State Key Laboratory of Synthetical Automation for Process Industries, Shenyang 110819, \\ China \\ *Corresponding author: zhaoyong@ise.neu.edu.cn
}

\begin{abstract}
This review covers photonic crystal cavities (PCCs) and their applications in optical sensors, with a particular focus on the structures of different PCCs. For each kind of optical sensor, the specific measurement principle, structure of PCC, and the corresponding sensing properties are all presented in detail. The summary of the reported works and the corresponding results demonstrate that it is possible to realize miniature and high-sensitive optical sensors due to the ultra-compact size, excellent resonant properties, and flexibility in structural design of PCCs. Finally, the key problems and new directions of PCCs for sensing applications are discussed.
\end{abstract}

Keywords: Photonic crystal cavity (PCC); optical sensor; high sensitivity; miniature sensor.

\section{Introduction}

Since E. Yablonovitch and S. John first proposed the concept of photonic crystal (PC) in 1987 $[1,2]$, PC, which possesses a periodic dielectric structure and the capability of guiding and manipulating light at the scale of optical wavelength, has been studied extensively both in theory and experiment $[3,4]$. One of the basic properties of PC is the photonic band gap (PBG), and the propagation of light within the frequency range of PBG will be forbidden [5]. Nevertheless, the periodicity of this dielectric structure will be broken when some defects are introduced in PC, which makes it possible for PC to present strong electromagnetic field confinement, small mode volume, and low extinction loss [6]. On the other hand, by adjusting the structural parameters of PC or infiltrating suitable materials in the air holes of PC, the propagation of light can be modified and engineered at will. Therefore, many PC based devices have been widely used in the applications of light flow control, such as filters [7, 8], electro-optical modulators [9, 10], switches [11, 12], and delay devices [13]. Specially, PC based sensors seem to be much more popular due to their promising characteristics like ultra-compact size, high measurement sensitivity, flexibility in structural design, and more suitable for monolithic integration [14-16]. Besides, the PC based sensors can also inherit the favorable characteristics of optical sensors, such as safety in flammable explosive environment, immunity to electromagnetic interference, long-distance monitoring, and rapid response speed. Therefore, during the last decades, many excellent optical sensors based on PC have been investigated and developed in a large range of sensing applications, such as gas sensors [17-19], liquid sensors [20], temperature sensors [21], stress sensors [22], refractive index (RI) sensors [23, 24], humidity sensors [25-26], and biochemical sensors [16, 27]. 
As a typical structure type, PC cavity (PCC) is formed by introducing point defects in the orderly arranged lattices. It exhibits strong spatial and temporal light confinement and long photon lifetime (namely, high quality factor $Q$ ) [28], thus greatly enhance the interaction strength between optical field and material of defected region. As for sensing applications, the enhanced interaction effect gives rise to an optical mode of PCC with a resonant wavelength that is highly sensitive to the local variations in its surrounding medium, and make PCC a promising building block for high-sensitive optical sensors [29]. In addition, the effective sensing area of PCC is on the order of a micrometer or less across, which provides an advanced sensing platform for in-situ monitoring with smart design.

In this work, an overview of optical sensors based on PCCs is introduced in detail, wherever available, to give a new perspective for further research on other sensing applications of PCCs. The rest of this paper is organized as follows: In section 2, the optical properties of PCCs and their basic sensing principles are analyzed and discussed. In section 3, the optical sensors based on PCCs, along with their structures and sensing properties, are presented. In section 4 and section 5 , the key problems and new directions of PCCs for sensing applications are put forward, respectively. Finally in section 6 , we draw a brief conclusion and prospect.

\section{Optical properties and sensing principles of PCCs}

From the view of defected structure, PCC can be divided into Ln ( $n \geq 3$ ) cavity [30-32], $\mathrm{H} m$ $(m=0,1,2)$ cavity [33, 34], mode-gap cavity [35], ring cavity [36, 37], and shoulder-coupled cavity $[38,39]$, as shown in Fig. 1.

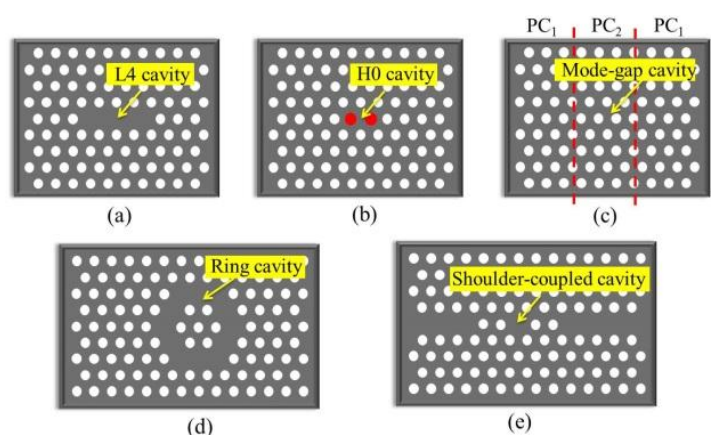

Fig. 1 Schematic structures of (a) L4 PCC, (b) H0 PCC, (c) mode-gap PCC, (d) ring PCC, and (e)

shoulder-coupled PCC.

Taking the shoulder-coupled cavity that published in Ref. [39] as an example, we will introduce the resonant properties and sensing principle of this PCC. To begin with, we analyze the property of PC waveguide (PCW), which is formed by removing the central row of air holes from the perfect PC along $x$ direction, as shown in Fig. 2(a), where $a$ is the lattice constant, $r$ is the radius of air hole, $d$ is the waveguide width, and $h$ is the slab thickness. Its basic property is that the light located in PBG can only be guided in the line waveguide as the light is confined horizontally by PBG of PC and vertically by total internal reflection due to the RI differences between different layers. Fig. 2(b) shows the calculated electric field distribution of PCW when the working frequency of transmission light is located in the PBG, which is simulated by using MIT's freely available software MEEP [40]. It is found that the TE-like polarized light can be strongly confined in waveguide region both in-plane direction (horizontally) and out-plane direction (vertically), and the leakage of light is very small. However, when the four air holes are introduced at the center of the above waveguide to form a shoulder-coupled cavity, most of the light energy will strongly 
localized in the central part of the PCC, as shown in Fig. 3(a). The corresponding transmission spectra of W1 PCW and shoulder-coupled PCC are shown in Fig. 3(b), from which we can find that the PCW possesses high transmittance and wide working range, while the transmission spectrum of PCC behaves a very narrow Lorentzian curve with a certain resonant frequency. Namely, only the light energy at resonant frequency could leak out from the central point defect.

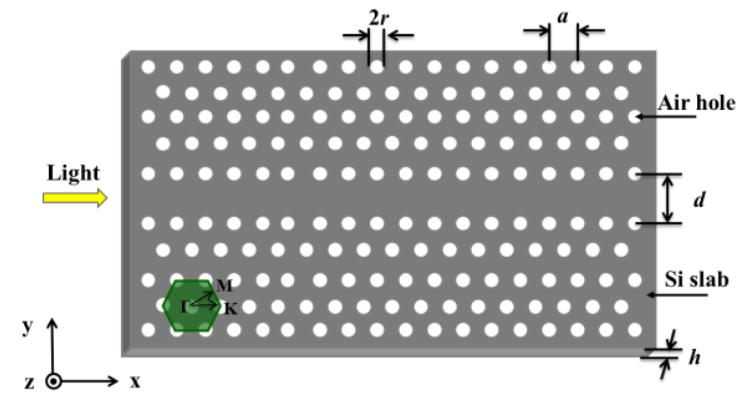

(a)

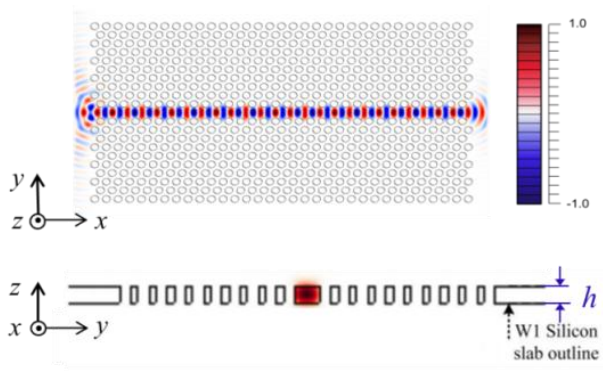

(b)

Fig. 2 Schematic structure of PCW (a) and its corresponding electric field distribution (b) ${ }^{[36]}$.

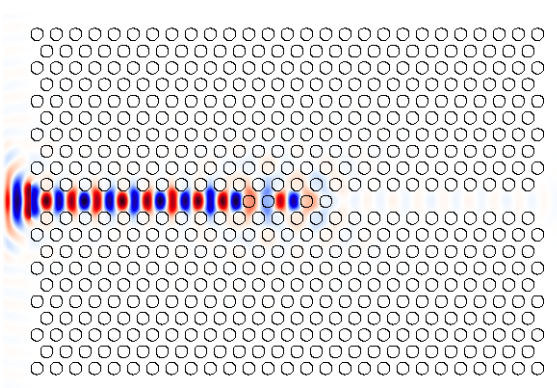

(a)

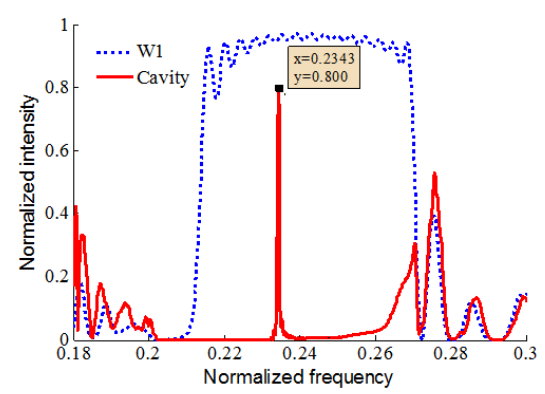

(b)

Fig. 3 (a) Electric field distribution of a shoulder-coupled PCC, and (b) transmission spectra of W1 PCW and shoulder-coupled PCC ${ }^{[36]}$.

It was demonstrated that the relationship between the normalized transmission intensity $T$ of shoulder-coupled PCC and working frequencies $\omega$ can be expressed approximately as a Lorentzian function [39]:

$$
T\left(\omega, \omega_{0}\right)=\frac{\left(\omega_{0} / 2 Q\right)^{2}}{\left(\omega-\omega_{0}\right)^{2}+\left(\omega_{0} / 2 Q\right)^{2}}
$$

where $\omega_{0}$ is the resonant frequency, $Q$ is the quality factor of this PCC and can be given as:

$$
Q=\left(\frac{1}{Q_{\omega}}+\frac{1}{Q_{r}}\right)^{-1}=\frac{Q_{\omega} Q_{r}}{Q_{\omega}+Q_{r}}
$$

where $Q_{\omega}$ is the lifetime of light to decay from the cavity into the waveguide, and $Q_{r}$ is the lifetime of light to radiate from cavity into the surrounding air.

Due to the unique properties of strong field confinement and high $Q$ factor of PCC, the resonant wavelength of PCC is highly sensitive to the ambient variations. Specially, the resonant wavelength $\lambda_{0}\left(\lambda_{0}=a / \omega_{0}\right)$ of PCC will shift with the RI variation of defected holes of PCC, as shown in Fig. 4. Somewhat like the Fabry-Perot cavity, the shift of resonant wavelength satisfies:

$$
\Delta \lambda_{0}=S \cdot \Delta n
$$

where $S$ is nearly constant when the resonant wavelength changes within a small range, and it is 
also the measurement sensitivity of PCC based RI sensor. If some other parameters, such as biochemical molecule, gas concentration, and mechanical effect, are invaded to change the RI of defected holes, they can also induce the shift of resonant wavelength. Thus, various high-sensitive and ultra-compact optical sensors based on PCCs are designed and proposed [33, 36, 38, 39].

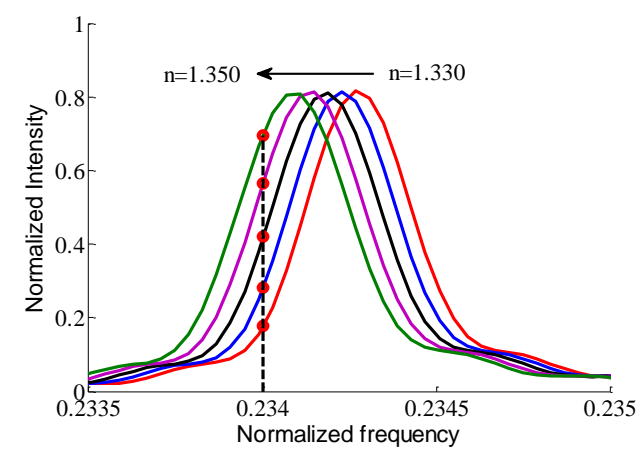

Fig. 4 Transmission spectra of PCC when the RI of defected holes is changed from 1.330 to 1.350 with an interval of $0.005^{[36]}$.

In addition, the detection limit $(C)$ of the PCC based RI sensor can be calculated by the measurement sensitivity $(S)$ and the minimal resolvable wavelength shift of PCC $\left(\Delta \lambda_{\min }\right)$, and it can be given by: $C=\Delta \lambda_{\min } / S$. For PCC, the relationship between $\Delta \lambda_{\min }$ and quality factor $Q$ follows: $\Delta \lambda_{\min }=\lambda_{0} /(10 Q)[41,42]$. Therefore, we have:

$$
C=\lambda_{0} /(10 Q S)
$$

From this equation, we can see that high RI sensitivity and high quality factor are required for $\underline{\mathrm{PCC}}$ to improve the sensing properties of PCC based sensors.

\section{Optical sensors based on PCCs}

\subsection{Refractive index sensor}

As discussed above, PCC exhibits strong field confinement and has long photon lifetime, which give rise to an optical mode with a resonant wavelength that is highly sensitive to $\underline{\mathrm{RI} \text { perturbation }}$ attributed to medium that infiltrated in the air holes of PCC, and thus allows us to implement various RI sensors based on PCC [43-49]. The measurement of RI change of glycerol-water mixture by monitoring the resonant wavelength shifts in various ratios was first demonstrated in 2004 by E. Chow et al. [43]. The proposed PCC was formed by reducing the radius of one central hole, and had a $Q$ factor of about 400. For RI measurement, the proposed ultra-compact sensor (sensing area of about $10 \mu \mathrm{m}^{2}$ ) demonstrated a measurement sensitivity of $200 \mathrm{~nm} / \mathrm{RIU}$ (refractive index unit) and detection limit of 0.002 RIU. Besides, it was concluded that the detection limit can be further improved by increasing the measurement sensitivity and the $Q$ factor of PCC and reducing the noise level of the measurement. According to this principle, various optimized PCCs have been designed to further improve the sensing properties of RI sensor. Table I summarized the structural schematics of PCCs and their corresponding sensing properties.

At the same time, a new type of waveguide, i.e. slot photonic crystal waveguide (SPCW), was theoretically proposed and experimentally demonstrated [50]. It is a waveguide formed by opening a slot along the line waveguide of PCW, and it has the unique characteristic of guiding and confining light in the low RI narrow slot with strong field enhancement [51]. Due to the electric-field discontinuity and high RI difference at the interface of silicon and low RI slot, the 
cavity mode inside the slot can be greatly enhanced [52]. In 2008, T. Yamamoto et al. [53] proposed a novel PCC in SPCW, in which the cavity was formed by locally modifying a few of air holes that adjacent to the waveguide. The simulation results demonstrated that the high $Q$ factor as high as $2 \times 10^{5}$ could be obtained. Then in 2009, Di Falco et al. [54] have experimentally demonstrated that the high $Q$ factor of up to 50000 and strong RI sensitivity of $1500 \mathrm{~nm} / \mathrm{RIU}$ could be obtained in the slot PCC, which was formed by varying the pitch of the surrounding PC along the slot waveguide axis. Later on, some other slot PCCs [55, 56] were proposed and demonstrated for their better applications in refractive index sensors, and their corresponding sensing properties are also summarized in Table I.

Table I Comparison of different PCCs that used for RI sensors and their sensing properties.

\begin{tabular}{|c|c|c|c|c|c|c|}
\hline Reference & $\begin{array}{l}\text { Schematic } \\
\text { structure }\end{array}$ & $\begin{array}{l}\text { Quality } \\
\text { factor }\end{array}$ & $\begin{array}{r}\text { Sensitivity } \\
\text { (nm/RIU) }\end{array}$ & $\begin{array}{c}\text { Detection limit } \\
\qquad C\left(\mathrm{RIU}^{-1}\right)\end{array}$ & $\begin{array}{c}\text { Experiment/ } \\
\text { simulation }\end{array}$ & $\begin{array}{c}\text { Published } \\
\text { year }\end{array}$ \\
\hline [43] & 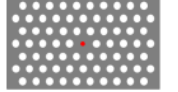 & 400 & 200 & 0.002 & Experiment & 2004 \\
\hline [44] & & 3820 & 330 & 0.001 & Simulation & 2008 \\
\hline$[45]$ & 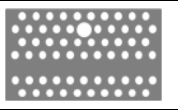 & 400 & 155 & 0.018 & Experiment & 2008 \\
\hline [45] & 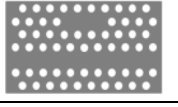 & 3000 & 63 & 0.006 & Experiment & 2008 \\
\hline [46] & & 17890 & 500 & 0.0001 & Simulation & 2013 \\
\hline [47] & $\begin{array}{l}\because \because \because 8: \\
\because \because 8 \% \\
\because \because \because 8 \% \\
\end{array}$ & 2966 & 131.7 & $3.797 \times 10^{-6}$ & Simulation & 2014 \\
\hline$[48]$ & 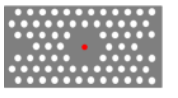 & $10^{7}$ & 330 & $1.24 \times 10^{-5}$ & Simulation & 2014 \\
\hline [49] & 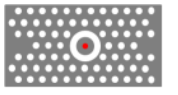 & $10^{7}$ & 160 & $8.75 \times 10^{-5}$ & Simulation & 2015 \\
\hline [54] & 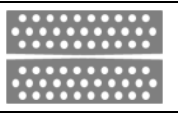 & 50000 & 1500 & $7.8 \times 10^{-6}$ & Experiment & 2009 \\
\hline$[55]$ & 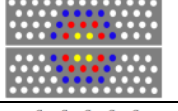 & 7500 & 370 & $2.3 \times 10^{-5}$ & Experiment & 2013 \\
\hline [56] & 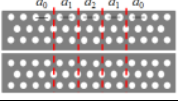 & 25000 & 235 & $1.25 \times 10^{-5}$ & Experiment & 2014 \\
\hline
\end{tabular}

The above results have demonstrated that PCCs with strong optical confinement and high $Q$ can be well used for RI measurement, along with the wavelength shift of resonant peak. The measurement results can also provide guidance for some other sensors, such as biosensors, chemical sensors, mechanical sensors and gas sensors, in which the change of these measurement parameters can all be converted into RI variations. As for RI sensor, there are also some other optical systems, such as surface plasma resonance (SPR) [57], modal interference [58], evanescent wave [59], and Fabry-Perot (F-P) cavity [60]. In Table II, we have summarized the best values of detection limit and compared the advantages and disadvantages of these optical systems for their applications in refractive index measurement. 
Table II Comparison of the presented optical systems that used for RI measurement

\begin{tabular}{|c|c|c|c|c|c|}
\hline $\begin{array}{l}\text { Optical } \\
\text { system }\end{array}$ & $\begin{array}{l}\text { Detection } \\
\text { limit } \\
\left(\text { RIU }^{-1}\right)\end{array}$ & $\begin{array}{r}\text { Sensitivity } \\
(\mathrm{nm} / \mathrm{RIU})\end{array}$ & Advantages & Disadvantages & Ref. \\
\hline PCC & $7.8 \times 10^{-6}$ & 1500 & $\begin{array}{l}\text { - Compactness; } \\
\text { - Integration; } \\
\text { - Easy to demodulate; } \\
\text { - Flexible in structural } \\
\text { design. }\end{array}$ & $\begin{array}{l}\text { - Large coupling loss; } \\
\text { - Temperature cross-sensitivity; } \\
\text { - Difficulty in fabrication. }\end{array}$ & {$[54]$} \\
\hline SPR & $5 \times 10^{-6}$ & 2000 & $\begin{array}{l}\text { - High sensitivity; } \\
\text { - Good flexibility and } \\
\text { extensibility. }\end{array}$ & $\begin{array}{l}\text { Difficult to fabricate; } \\
\text { - Temperature cross-sensitivity; } \\
\text { - Working in non-communication } \\
\text { wavelengths (most). }\end{array}$ & {$[57]$} \\
\hline $\begin{array}{c}\text { Modal } \\
\text { interference }\end{array}$ & $1.74 \times 10^{-6}$ & 580 & $\begin{array}{l}\text { - Low cost; } \\
\text { - Easy to fabricate; } \\
\text { - Simple structure. }\end{array}$ & $\begin{array}{l}\text { - Temperature cross-sensitivity; } \\
\text { - Interferences of multiple modes } \\
\text { (Non-linear output). }\end{array}$ & {$[58]$} \\
\hline $\begin{array}{c}\text { Evanescent } \\
\text { wave }\end{array}$ & $10^{-6}$ & 700 & $\begin{array}{l}\text { Good flexibility and } \\
\text { extensibility. }\end{array}$ & $\begin{array}{l}\text { - Lack of robustness; } \\
\text { - Low transmittance; } \\
\text { Influence to light intensity } \\
\text { fluctuations. }\end{array}$ & [59] \\
\hline F-P cavity & $1.64 \times 10^{-5}$ & 670000 & $\begin{array}{l}\text { - Low cost; } \\
\text { - Simple structure. }\end{array}$ & $\begin{array}{l}\text { - Uneasy to control cavity length; } \\
\text { - Temperature cross-sensitivity; } \\
\text { Lacks of flexibility and } \\
\text { extensibility; } \\
\text { - Difficult to demodulate. }\end{array}$ & {$[60]$} \\
\hline
\end{tabular}

\subsection{Biochemical sensor}

As the concentration of biochemical sample is directly linked to the RI of target analyte, now the commercially available biochemical sensors usually exploit the RI change induced by the analyte interaction with the optical field as the sensing mechanism. Through this means, the target analytes can be detected in their natural forms without any modifications. Along with the development of PCC based RI sensors, many PCC based biochemical sensors were subsequently proposed and demonstrated. In 2005, the measurements of cation and anion concentrations were demonstrated by using a L4 PCC coated with ion-selective polymer [61]. Besides, it was showed that increasing the length of cavity would enhance the $Q$ of cavity by an order of magnitude and then would improve the shift of resonant wavelength while retaining compact size characteristic $[62,63]$. Then in 2007, it was reported that a PCC resonator with only one spot defect could able to detect protein molecule as small as $2.5 \mathrm{fg}$, while the active sensing volume could be down to $0.15 \mu \mathrm{m}^{2}$ [64]. Besides, this structure could also be used to detect a gold nanoparticle with $10 \mathrm{~nm}$ in diameter [65] and anti-biotin with concentration of $20 \mathrm{pM}$ (corresponding to less than $4.5 \mathrm{fg}$ of bound material on the sensor surface and fewer than 80 molecules in the modal volume of the cavity) [66]. Latter in 2010, F. L. Hsiao et al. [67] first demonstrated that the ring PCC (see Fig. 5(a)) could also be used for high-sensitive monitoring of reaction kinetics and protein concentration with the minimum detectable biomolecule weight of only $0.2 \mathrm{fg}$, which showed 
promising applications when the detection of biomolecule down to the level of single copy of DNA was needed. In this design, the ring PCC was formed by integrating terminal waveguides, i.e., line defects, and a hexagonal ring waveguide, i.e., a hexagon trace defect, in a $220 \mathrm{~nm}$ thick device layer of silicon wafer. The lattice constant was defined as $410 \mathrm{~nm}$ and the radius of holes was set as $120 \mathrm{~nm}$. In addition, when two ring PCCs were cascaded together (see Fig. 5(b)), it would be possible to detect two kinds of target DNA molecules or realize a temperature compensated biosensor [68]. A major advantage of this structure was that it allows the measurements of multiple biomolecules at the same input port through the use of appropriate sensing holes and offers the possibility to implement the corroboration mechanism by exchanging

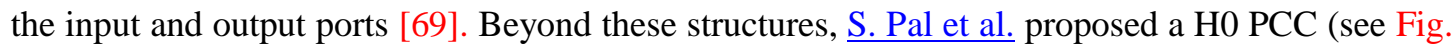
5(c)), which could be used for the measurement of $\operatorname{lgG}$ molecule with detection limit of $1.5 \mathrm{fg}$ [70] and Human Papillomavirus virus-like particles (VLPs) with detection limit of $1.5 \mathrm{nM}$ [71]. In the design, the defect hole had a radius of $0.2 a$, and the surrounding air hole radii were fixed at $0.3 a$. As each PCC sensor could potentially be functionalized with different receptor molecules, it was also possible to detect multiple pathogenic viruses, or, alternatively, different strains of the same virus, on the same chip. Combined the spatial confinement of optical field provided by slot waveguide with the temporal confinement of optical field in PCC, M. G. Scullion et al. [72] first demonstrated the possibility of slot PCC (see Fig. 5(d), lattice constant $490 \mathrm{~nm}$, cavity period 460 nm, hole radius $135 \mathrm{~nm}$ and slot width $120 \mathrm{~nm}$ ) in the detection of dissolved avidin concentration as low as $15 \mathrm{nM}$, with the sensing area of only $2.2 \mu \mathrm{m}^{2}$.

From the above results, we can find that with the appropriate choice of receptor that infiltrated in the air holes of PCC, the PCC sensor could be adapted to detect any biochemical molecules, whose performances can be further improved by designing cavities with higher $Q$ factors and by localizing the target molecular recognition processes in the defect region. The small size of the device, combined with strong successful implementation of multiple PCCs, provides a strong potential for large arrays of independent sensors on a centimeter sized chip.

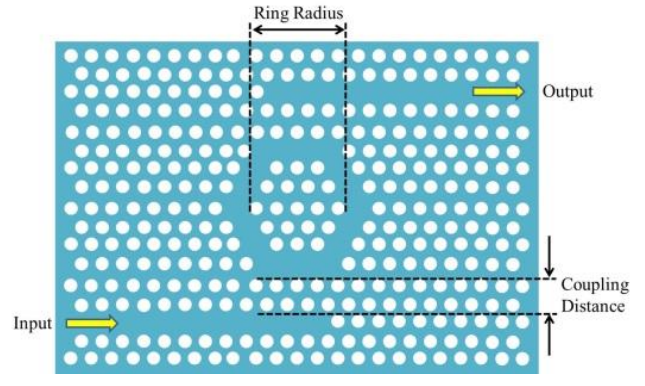

(a)

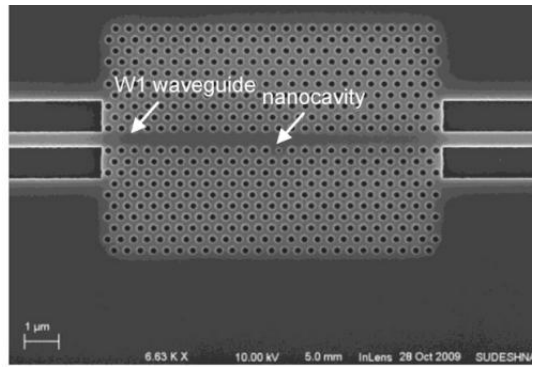

(c)

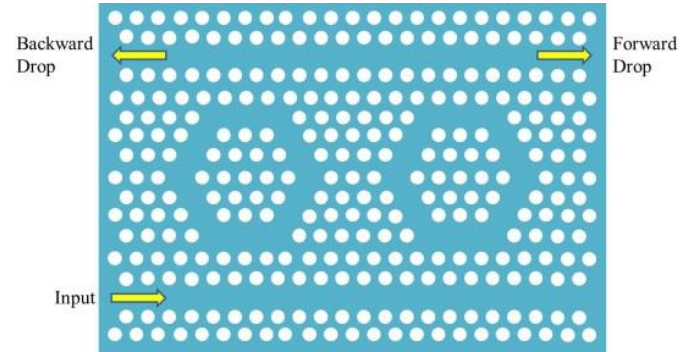

(b)

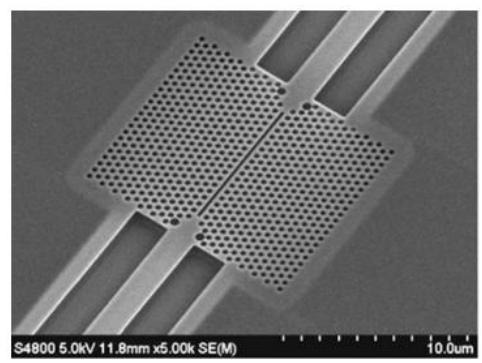

(d)

Fig. 5 Schematic structures of (a) ring PCC ${ }^{[60]}$ and (b) cascaded ring PCC ${ }^{[61]}$ that used for biochemical sensors, and SEM images of (c) H0 cavity ${ }^{[63]}$ and (d) slot PCC ${ }^{[65]}$ that used for biochemical sensors. 


\subsection{Gas sensor}

The periodic air hole microstructure of PCC is a natural candidate for housing gas analytes, thus, the resonant wavelength of PCC, as well as the RI of air hole, would change with the concentration variation of infiltrated gas or the variation of ambient pressure. This is also the measurement principle of gas sensor based on PCC. Comparing with the traditional optical gas sensor, the size of PCC based gas sensor could be drastically reduced.

One example is the measurement of gas concentration in a gas mixture of two gases with different refractive indices or relative gas pressures [73, 74]. In Ref. [73], a heterostructure PCC was formed by modulating the radii of the first row of air holes adjacent to the waveguide (see Fig. 6(a)), which resulted in a sensitivity of $80 \mathrm{~nm} / \mathrm{RIU}$ and quality factor of 380000. As shown in Fig. 6(b), this PCC structure could be well used to identify vacuum, nitrogen, and $\mathrm{SF}_{6}$. Besides, when the pressure for $\mathrm{SF}_{6}$ atmosphere was changed in a step of $0.5 \times 10^{4} \mathrm{~Pa}$, an obvious wavelength shift of the PCC would be observed as shown in Fig. 6(c). In this design, the radii of some holes were enlarged to $0.27 a$ along the W1 waveguide to create mirror regions (marked with A). The actual cavity (marked with $\mathrm{C}$ ) is enclosed between those mirror regions, whose radii were $0.25 a$. Between region $\mathrm{A}$ and region $\mathrm{C}$, the radii of the holes were increased linearly (region B).

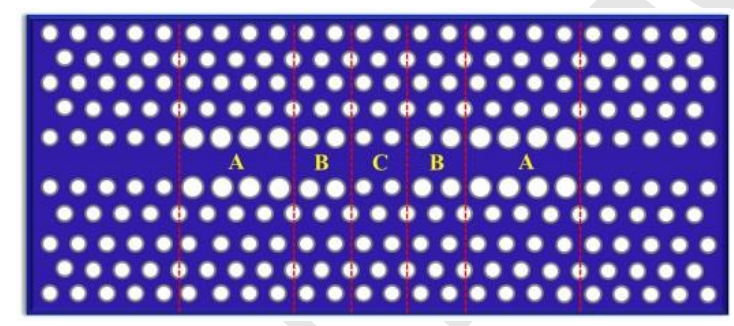

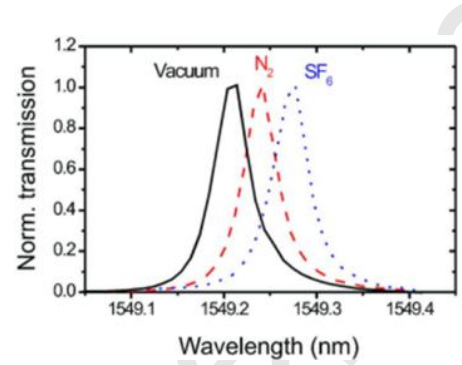

(b)

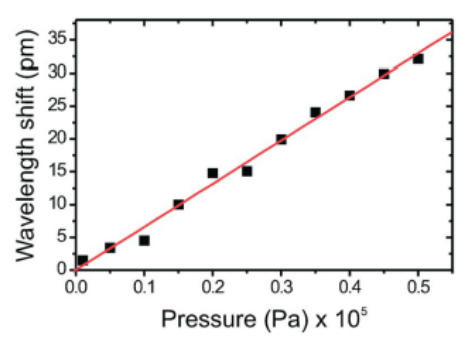

(c)

Fig. 6 Structure of heterostructure PCC (a), the corresponding transmission spectra for vacuum, nitrogen, $\mathrm{SF}_{6}$ gases (b), and the relationship between resonant wavelength shift and surrounding pressure (c) ${ }^{[66]}$.

Then in 2010, J. Jágerská et al. [74] improved the measurement sensitivity of gas RI by introducing a heterostructure slot PCC. The cavity length was defined to be $L=3 a$, and the resonant state of which was found between the cutoffs of the 120 and $100 \mathrm{~nm}$ wide slot waveguides, as shown in Fig. 7(a). As for the measurements of helium, nitrogen, and carbon dioxide, an experimental sensitivity up to $510 \mathrm{~nm} / \mathrm{RIU}$ and the detection limit higher than $1 \times 10^{-5}$ RIU were demonstrated. However, the heterostructure PCC needs to be carefully optimized and finely tuned in order to achieve ultrahigh $Q$, and as a result it has a low tolerance to fabrication deviations. Recently, K. Li et al. [75] proposed and experimentally demonstrated a series of Ln slot PCCs (see Fig. 7(b)), which operated as gas sensors. Finally, the quality factor exceeding $\underline{30000}$, sensitivity up to $421 \mathrm{~nm} / \mathrm{RIU}$, and detection limit down to $1 \times 10^{-5}$ RIU were experimentally demonstrated. The simple structure and high fabrication tolerance of this PCC extended its 
applications in optical sensors. But it should be mentioned that the RI of target gas is always small ( 1.0) and the corresponding RI variation due to concentration change is usually lower than $10^{-4}$ RIU, so the above methods cannot be used to identify the concentration of target gas. Besides, as any other gases and environment parameters can all result in the RI variation of air hole, they would bring many unpredictable errors to the measurement system, and even cause the system unable to work.

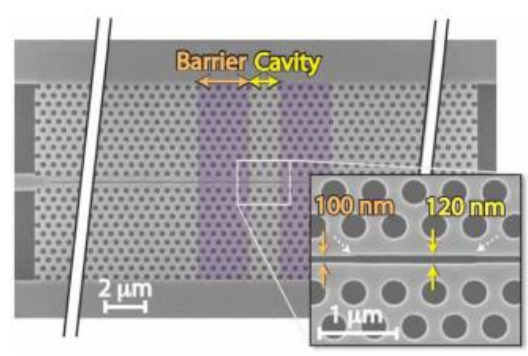

(a)

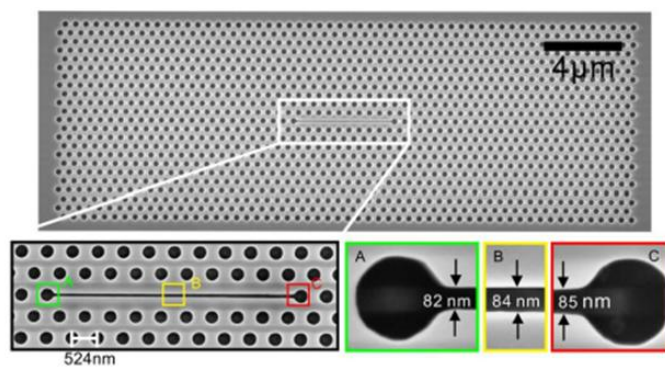

(b)

Fig. 7 Structures of heterostructure slot $\mathrm{PCC}^{[67]}$ and $\mathrm{L} n$ slot PCCs ${ }^{[68]}$ for gas sensing.

To resolve these problems and play the advantages of ultra-compact and high-sensitivity of PCC based gas sensor, Y. Zhang et al. [19] first proposed a gas concentration sensor with a cryptophane E infiltrated PCC (see Fig. 8(a)). In this design, the lattice constant was $a=351 \mathrm{~nm}$, the radius of bulk air hole was $r=0.3 a$, and the thickness of the PC slab was $h=0.6 a$, the defected radius was $r_{1}=0.45 a$. The concentration variation of methane would change the RI of cryptophane E that infiltrated in the defected holes of PCC, and then induce a shift of resonant wavelength, allowing precision measurement of methane concentration. By combing selective adsorption property of cryptophane E to methane and excellent resonant properties of PCC, the resonant spectrum of PCC would shift sharply with the concentration change of methane gas, as shown in Fig. 8(b). As a result, a theoretical detection limit of $697.35 \mathrm{ppm}$ for methane sensing could be achieved, which provides a new direction for the gas sensor based on PCC.

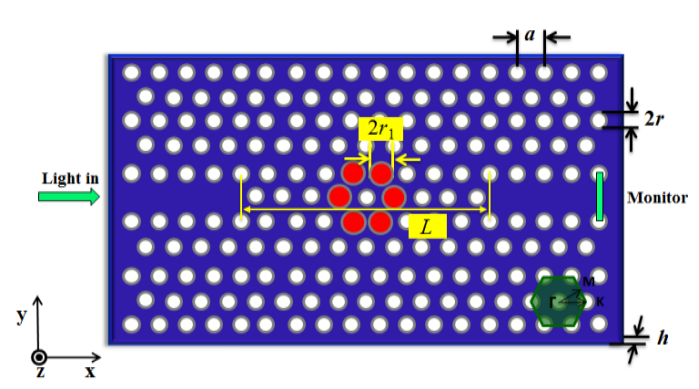

(a)

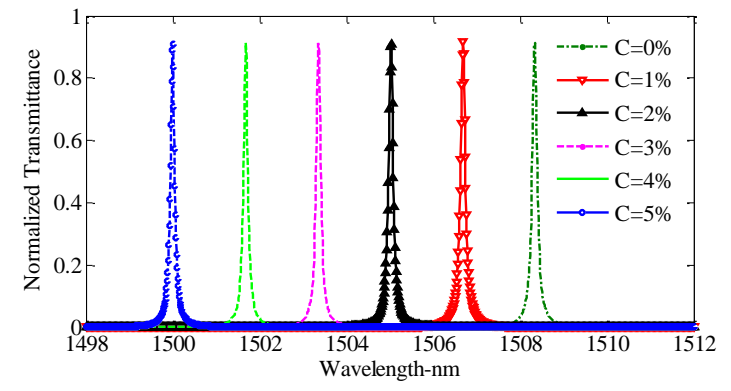

(b)

Fig. 8 Schematic structure and the corresponding transmission spectra of cryptophane E infiltrated PCC ${ }^{[69]}$.

\subsection{Mechanical sensor}

The operation principle of mechanical sensor based on PCC is the photoelastic, piezoelectric, and electrooptic effects of the materials constituting the PCC structure. When certain mechanical action is applied to the PCC, it will induce the effective RI variation, deformation, or deflection of the PCC structure, which will then modify the transmission spectrum of the PCC, and thus shift the corresponding resonant wavelength. The amount of such spectral shift can be therefore exploited to measure the applied mechanical action. 
In 2007, T. Stomeo et al. [76] proposed a H1 PCC for pressure sensor. The drop peak that corresponded to the resonant wavelength of the mode localized in the cavity shifted its spectral position with a linearity sensitivity of $5.82 \mathrm{~nm} / \mathrm{GPa}$ for pressure ranging between $0.25 \mathrm{Gpa}$ and 5 GPa. Considering the effective action area of the applied pressure was equal to $1 \mathrm{~mm}^{2}$, the detection limit of about $0.3 \mathrm{mN}$ could be obtained. Then, C. Lee et al. demonstrated that the shoulder-coupled PCC in a suspended silicon bridge structure could also be used for pressure measurement [77]. As shown in Fig. 9(a), the lattice constant was $500 \mathrm{~nm}$, the radius of all holes was $180 \mathrm{~nm}$, and the initial defect length was $640 \mathrm{~nm}$. Longitudinal deformation of air holes and a change in defect length of the cavity caused by applied pressure would all shift the resonant wavelength of PCC. It was concluded that the minimum detectable force and the minimum detectable vertical deflection were $0.25 \mathrm{~N}$ and $20-25 \mathrm{~nm}$, respectively. Besides, it was demonstrated that the shape change of the air holes in the deformed PCC structure has just a little effect on the output resonant behavior. More importantly, the relative position shift of these air holes in the deformed PCC plays a major role in contribution to the output resonant behavior. Upon this structure, C. Lee et al. investigated the mechanical property of this PCC, by using a silicon cantilever (see Fig. 9(b)) [78, 79]. In the graph of strain versus resonant wavelength shift, a rather linear relationship was observed even for different cantilevers. For a $30 \mu \mathrm{m}$ long and $15 \mu \mathrm{m}$ wide cantilever, the detection limits for stain, vertical deflection at the cantilever end, and force load were $0.0133 \%, 0.37 \mu \mathrm{m}$, and $0.0625 \mathrm{~N}$, respectively. Latter in 2011 [80, 36], they further investigated silicon cantilever with ring PCC as the mechanical sensor. The ring PCC was formed by a regular hexagonal array of air holes with lattice constant of $410 \mathrm{~nm}$ and holes radii of $120 \mathrm{~nm}$. In Ref. [80], it was demonstrated that the sensing sensitivity of ring PCC (see Fig. 10(a)) could be greatly improved when compared with shoulder-coupled PCC. Finally, the minimum detectable force and strain for ring PCC were $75.7 \mathrm{nN}$ and $0.0023 \%$, respectively. Then in Ref. [36], two ring PCCs were integrated on the silicon cantilever (see Fig. 10(b)) for mechanical sensing. By investigating the sensing characteristics of dual ring PCCs at various positions adjacent to the junction of the cantilever and the substrate, the minimum detectable force as low as $7.58 \mathrm{nN}$ was obtained. In the same year, they proposed circular Si diaphragm integrated with triple ring PCC (see Fig. 10(c)) as the mechanical sensor [81], which given minimum detectable force of $0.847 \mu \mathrm{N}$ in the wide force range of $10 \mu \mathrm{N}$ to $20 \mu \mathrm{N}$.

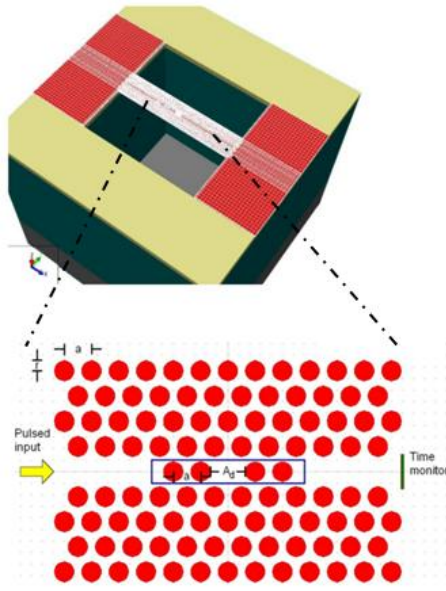

(a)

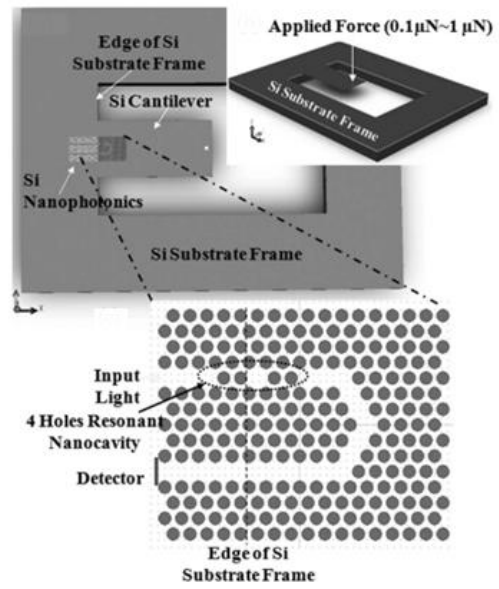

(b)

Fig. 9 Structures of shoulder-coupled PCC in (a) suspended silicon bridge ${ }^{[71]}$ and (b) silicon cantilever ${ }^{[72]}$ for mechanical sensing. 


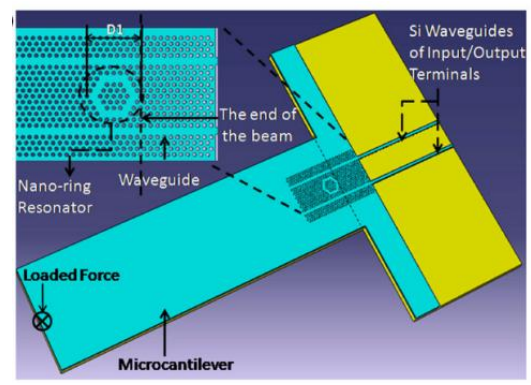

(a)

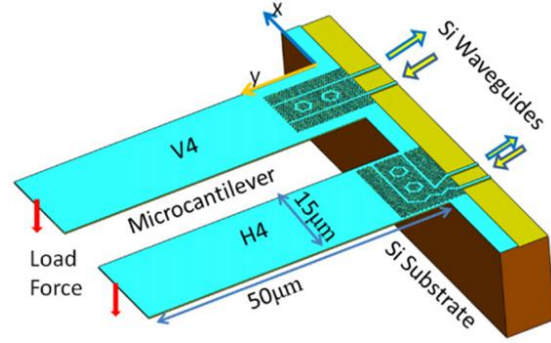

(b)

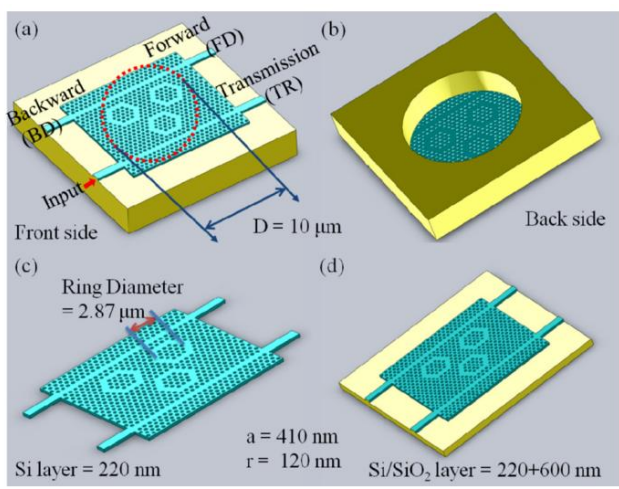

(c)

Fig. 10 Structures of one ring PCC ${ }^{[74]}$, dual ring PCC ${ }^{[33]}$, and triple ring PCC ${ }^{[75]}$ for mechanical sensing.

At the same time, many different PCC structures were proposed by some other research teams to study the mechanical properties [82-84]. In Ref. [82], B. T. Tung et al. demonstrated that the theoretical minimum detectable strain for a L3 PCC was 8.5 nع. In Ref. [83], D. Yang et al. studied the properties of H0 slot cavity (see Fig. 11(a)). With the structural parameters of radius $r=0.32 a$,

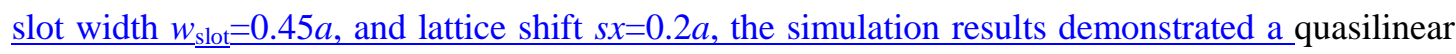
measurement of microdisplacement with a sensitivity of $1.0 a^{-1}$. In Ref. [84], D. Mao et al. realized the minimum surface stress of $0.8 \mathrm{mN} / \mathrm{m}$ by integrating a cantilever inside a PCC (see Fig. 11(b)).

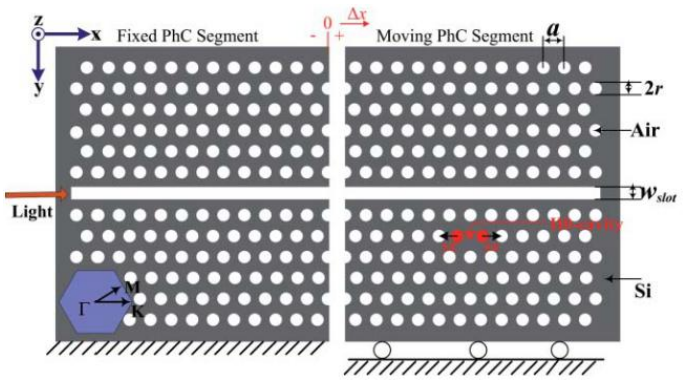

(a)

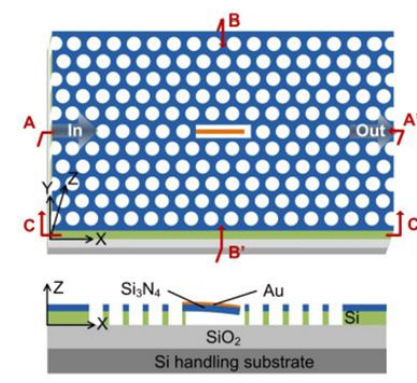

(b)

Fig. 11 (a) Structure of H0 slot cavity for displacement sensing ${ }^{[77]}$ and (b) structure of PCC integrated with cantilever for stress sensing ${ }^{[78]}$.

And in Ref. [38], Y. Yang et al. proposed a shoulder-coupled PCC, as shown in Fig. 12(a), whose lattice constant was $385 \mathrm{~nm}$, radius of air holes was $0.3 a$, thickness was $0.56 a$, and shift of holes was $0.2 a$. Simulation results demonstrated that the proposed PCC could be used for the stress detection in both horizontal and vertical directions, with detection limits of $58 \mathrm{nN}$ and 44 $\mathrm{nN}$, respectively. However, if the mechanical actions are too large, the deformation or deflection of PC lattice structure will be serious [38]. As a result, the resonant conditions (such as shape of 
resonant spectrum, $Q$ factor, and transmittance) of PCC will be changed, which will influence the sensing properties (sensitivity, linearity, detection limit, stability, sensing range, etc.) of the mechanical sensor and even cause the sensor unable to work [77]. To resolve this problem, in 2013, D. Yang et al proposed a torsion-free pressure sensor based on a piston-typed H0 cavity structure, as shown in Fig. 12(b) [85]. Here, the H0 cavity was achieved by adding a nanohole in the center of defected hole. In the design, the radius of air holes was $r=0.32 a$ and the lattice constant was $a=425 \mathrm{~nm}$. Finally, the sensitivity as high as $0.50 \mathrm{~nm} / \mathrm{nN}$ was observed and the detection limit was estimated to be as small as $0.68 \mathrm{nN}$. Combining the advantages of shoulder-coupled PCC in Ref. [38] and piston-typed H0 PCC in Ref. [85], Y. Yang proposed a three dimensional force sensor (see Fig. 12(c)) [86], in which the shoulder-coupled PCC was used to detect the forces in the horizontal and vertical directions, and the piston-typed H0 PCC was used to detect the force in the upright direction. Finally, by designing and optimizing this novel

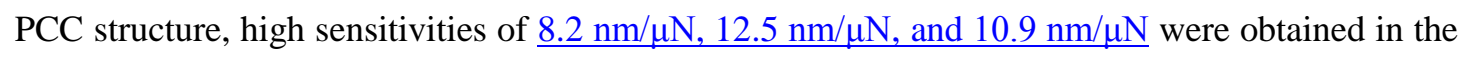
horizontal direction, vertical direction and upright direction. Correspondingly, the detection limits for three directions were $24 \mathrm{nN}, 16 \mathrm{nN}$, and $18 \mathrm{nN}$, respectively. This novel sensing mechanism creates a new vision of PCC based mechanical sensors.

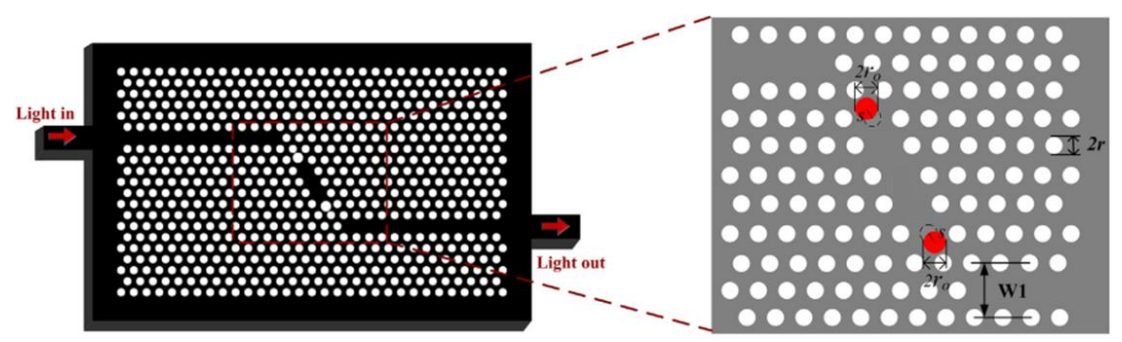

(a)

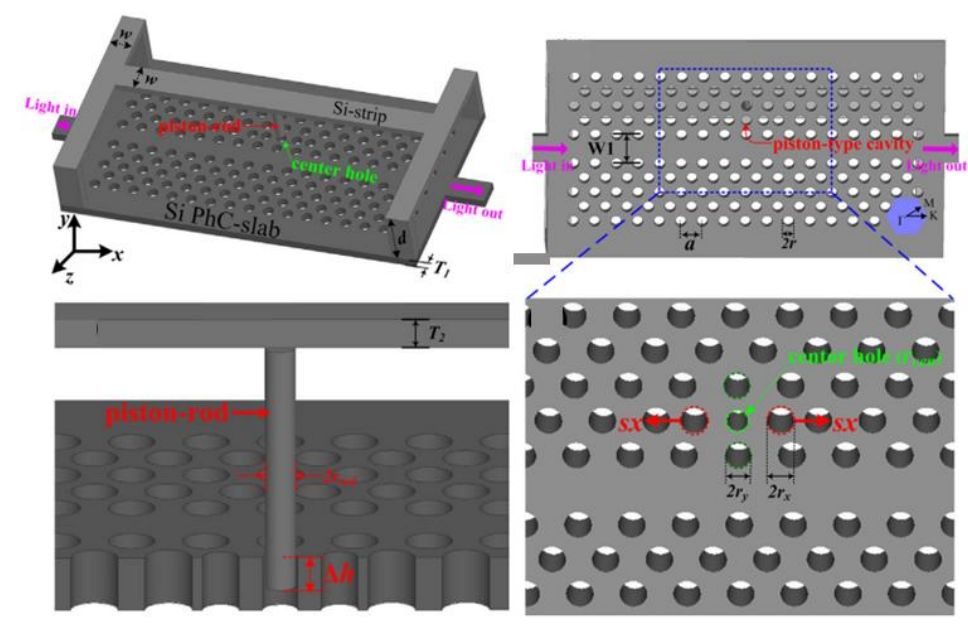

(b)
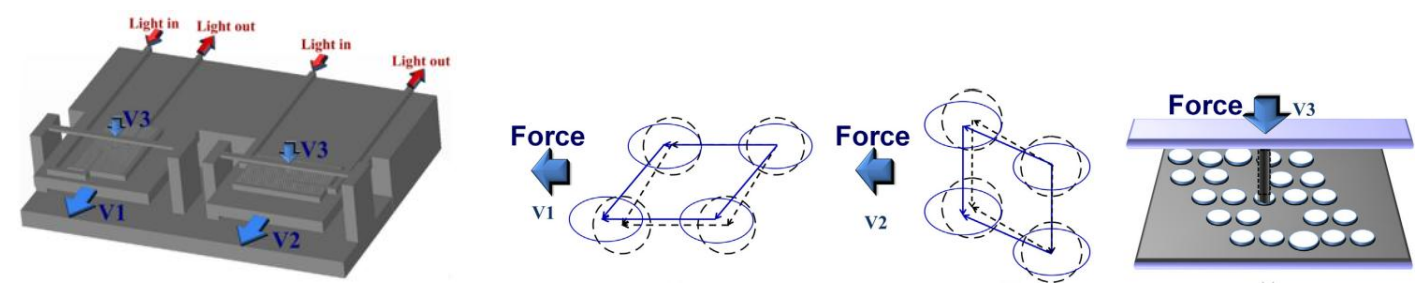

(c)

Fig. 12 Structures of shoulder-coupled PCC ${ }^{[35]}$, piston-typed PCC ${ }^{[79]}$, and modular PCC ${ }^{[80]}$ for force sensing. 


\subsection{Other sensors}

In addition to the above sensing applications, the PCC can also been used for electric voltage sensor based on electro-optical effect of polymer and magnetic field sensor based on magneto-optical effect of magnetic fluid. In 2011, D. Yang et al. [87] proposed an electro-optic sensor by using a $\mathrm{H} 0$ cavity infiltrated with nonlinear optical polymer. As shown in Fig. 13(a), the triangular lattice PC was realized on a silicon slab with air holes infiltrated with polymer $\left.\underline{n}_{\text {polymer }}=1.6\right)$, and having lattice constant of $403 \mathrm{~nm}$, radius of $128.96 \mathrm{~nm}$, slab thickness of 221.65 $\mathrm{nm}$, and lattice shift of $80.6 \mathrm{~nm}$. Besides, there were two micro-electrodes that were placed on each side of PC slab. If the driving voltage varied, the RI of polymer would be changed accordingly because of the Pockel's effect, which would in turn shift the resonant drop of H0 cavity, as shown in Fig. 13(b). Finally, the linear measurement sensitivity of $31.9 \mathrm{~nm} / \mathrm{V}$ was obtained. Coincidentally, in 2015, Y. Zhao et al. [88] demonstrated a magnetic field sensor by using a cascaded $\mathrm{H} 0$ cavity infiltrated with magnetic fluid. As shown in Fig. 14, two PC cavities with $r_{1}=0.32 a$ and $r_{2}=0.30 a$ were integrated on a monolithic silicon substrate and side-coupled to a single PCW to form cascaded PC cavities, in which the two defected regions were infiltrated with two different types of magnetic fluid. The two resonant dips of two PC cavities were independent of each other, and a shift in one of them did not perturb the other. This allowed the implementation of two individual sensors under the same environment, and eventually realized the simultaneous measurement of magnetic field and temperature. The implication which we can draw from the above two sensors is that the application field of PCC based sensor can be further extended by infiltrating some sensitive materials into air holes of PCC.
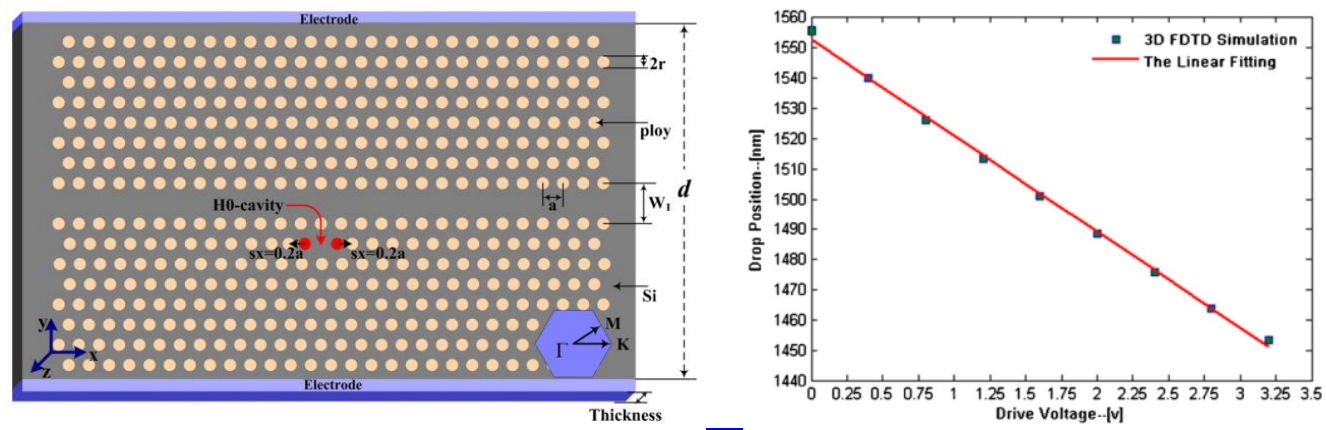

Fig. 13 Schematic structure and the corresponding sensing property of polymer infiltrated PCC ${ }^{[87]}$

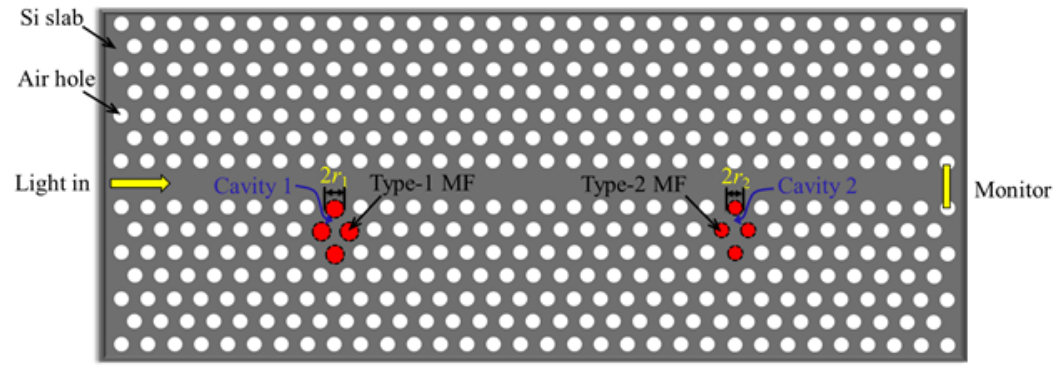

Fig. 14 Schematic structure cascaded H0 PCC with magnetic field infiltration ${ }^{[88]}$.

\section{Key problems of PCCs for sensing applications}

Although the rapid development and great potential of PCC based optical sensor, there still exist some key problems for its practical applications, such as fabrication errors, coupling problem, and temperature influence. 


\subsection{Fabrication errors}

In actual implementations, the stability of resonant properties of PCC against fabrication errors is a significant factor. At the current technology of PC fabrication, the position and size of air hole can be controlled in the level of $1 \mathrm{~nm}$ [89] and 2-4 nm [90], respectively. D. Pergande et al. [91] demonstrated that the overall transmission of bulk PC was limited by fluctuations of the holes radii. Besides, a pore radius fluctuation of $1 \%$ would lead to the attenuation in the transmission of $15 \mathrm{~dB} / \mathrm{mm}$. Since the interaction strength between optical field and materials of defected region is relatively strong, the fabrication errors of cavity region of $\mathrm{PCC}$ will have much more significant impacts on the resonant properties of PCC. For example, H. Hagina et al. [92] demonstrated that the ultimate $Q$ of heterostructure PC cavity would reduce to one eighth of ideal one when only 1 nm error was introduced to the holes radii. Besides, the fabrication errors of PCW are complicated, random, unpredictable, and immeasurable, so how to ease of fabrication and obtain achievable tolerances are important considerations in future.

\subsection{Coupling problem}

Before the practical applications of PCC, one subsequent challenge is the difficult to efficiently couple light from conventional single mode fiber into PCC device [63]. To decrease the coupling loss, the most common method is to introduce PCWs on both sides of PCC [38]. Then, the light is firstly emitted from conventional single mode fiber to PCW, and then transmitted through the $\underline{\text { PCW to PCC. However, it remains challenging because the typical waveguide cross-section }(<1}$ $\mu \mathrm{m}$ width and $<500 \mathrm{~nm}$ thickness) makes it difficult to couple light in and from the fiber core of conventional single mode fiber ( $8-10 \mu \mathrm{m}$ diameter). Additional difficulties are encountered due to the different transmission principles of PCW (based on PBG theory) and conventional single mode fiber (based on total internal reflection). In general, the coupling loss can be greatly decreased by accurate aligning of conventional fiber with the PC device through an adjustable mechanical device and proper designing of the coupling interface.

\subsection{Temperature influence}

As we know, the refractive index of silicon is thermal dependence, which means that the resonant properties of silicon based PCC device are easily influenced by external temperature [21]. To prohibit the undesired variation, precision temperature control system is essential in practical application, which will then add the size and cost of the PCC based sensors. This effect had not been taken into account in the previous researches until 2009, when C. Karnutsch et al. [93] proposed a temperature-insensitive PCC based on the optofluidic technology. By designing suitable dimensions and using a liquid with an appropriate thermo-optic coefficient, it was demonstrated that the thermo-optic effect of the infiltrated optofluidic could minimize or even eliminate the temperature dependence of the device for a desired working range. However, the utilization of optofluidic infiltrated PCC might also decrease the flexibility of PCC in structural design. Therefore, how to decrease the temperature influence is also a critical problem in future.

\section{New directions of PCCs for sensing applications}

\subsection{Optofluidic controlled PCC}

As discussed above, the sensing properties of PCC based sensors are in close relation with the $Q$ factor of PCC. Current methods to realize high- $Q$ PCCs typically rely on extremely precise control of size and position of air holes on the order of nanometer, both of which are hard to 
achieve precisely. Therefore, the nanometer-scale precision required to realize sophisticated and optimized structures eventually becomes a limiting factor in achieving high- $Q$ PCCs, as pointed out by C. Asano et al. [94]. Besides, the working wavelength of PCC can only be located at a certain value and cannot be varied once fabricated, which will limit the application situations and further developments of PCC based sensors.

Very recently, there is a new photonic branch to integrate nanophotonics on the manipulation of photons at the scale of optical wavelength with microfluidics on the control of fluids at the micron scale, which defines a major part of optofluidics field [95]. Besides, the infiltrated fluids possess a wide range of refractive indices (from 1.33 for water based solutions to 1.5 for silica oil matching fluids and to above 1.8 for Cargille fluids), which are especially effective in tuning photonic structures beyond that accessible through infiltration of solid materials. Demonstrations of optofluidic devices exploit the characteristics of fluids to achieve dynamic manipulation of optical properties and reveal the promise for their widespread use [96]. This provides a potential technology to realize high-sensitive optical sensors, and offers a flexible means to write, tune or reconfigure photonic devices for a swathe of applications [97]. In this regard, it has been shown that PCs in general and PCCs in particular can be advantageously exploited within optofluidic architectures [98-100]. In addition, both the amount of liquid and the location of the selectively infiltrated area can be accurately controlled by using an integrated optofluidic circuit bonded onto the lithographic masking [101], a confocal laser scanning microscope equipped with a micro-infiltration system [96], or a computer controlled micropipette [102] whose size is comparable to the air holes. By exploiting the inherent flexibility of fluid infiltration, the optofluidic infiltration schemes not only offer the potential for realizing tunable and reconfigurable PCCs at will with no need of structural variation, but also provide the flexibility to create spatially programmable PCCs according to practical requirements. In this case, the infiltration of fluids into the air holes of PCC devices has been popularly investigated and demonstrated.

In 2006, S. Tomljenovic-Hanic et al. [103] designed a heterostructure PCC (see Fig. 15(a)) without changes of any structural parameters. It was numerically demonstrated in Fig. 15(b) that the $Q$ factor value of this design achievable by substituting the air in the holes with polymer materials or liquid crystals was higher than $Q=9.7 \times 10^{5}$. This approach represents a novel technique for creating ultrahigh- $Q$ PCCs and furthermore opens the possibility of post-processing in PCCs. Then in 2007 [102], this method was experimentally investigated via evanescent probing from a tapered fiber at telecommunication wavelengths. Results demonstrated a PCC with $Q$ factor of 4300, which did not require nanometer-scale alterations in structural geometries and may be undertaken at any time after the PCC fabricated. The spectral and spatial reconfigurability of the proposed heterostructure PCCs were further demonstrated in 2008 [104], which showed high $Q$ factor $(\approx 10000)$ resonances for a broad range of cavity lengths. At the same time, $U$. Bog et al. [105] also demonstrated a post-processed heterostructure PCC by selective fluid infiltration of air holes using a glass microtip, which resulted in a higher intrinsic $Q$ factor of 57000 in experiment. Besides, A. C. Bedota et al. [106] have exploited the infiltration and evaporation dynamics of the liquid crystal within this heterostructure PCC by using a Fabry-Perot model that accounted for the joint effects of liquid volume reduction and cavity length variation due to liquid evaporation. It was demonstrated that the evaporation time is proportional to the volume-to-surface ratio, and therefore roughly scales with the linear dimension of the system. Besides the above 
heterostructure cavities, in 2009, F. Intonti et al. [107] studied the spectral tuning mechanism of point-defected PCC by controlled removal of locally infiltrated water. The micro-infiltration with water of one or few cavity holes and its subsequent controlled evaporation provided the possibility to local and continuous tune the cavity resonances in a spectral range larger than $20 \mathrm{~nm}$. Besides, it was also demonstrated that the addition of water in the microcavity region could improve its $\mathrm{Q}$ factor. And in 2012, N. W. L. Speijcken et al. [100] demonstrated the in situ optofluidic control of reconfigurable PCC, in which an extremely low vapour pressure oil was used to avoid evaporation issue at room temperature, and the infiltrated oil could be selectively removed from the defect by increasing the power of the excitation laser and systematically moving the focus position. Beyond that, C. Kamutsch et al. [93] have demonstrated that this post-fabrication technology can also be used for the temperature stabilization of PCC device. The key principle behind this optofluidic temperature stabilization was the concept that a substance with negative thermo-optic coefficient balanced the thermal drift of the host PC material. This temperature-stable cavity constitutes a major building block in the development of high-sensitive sensor systems for chemical and biomedical applications.

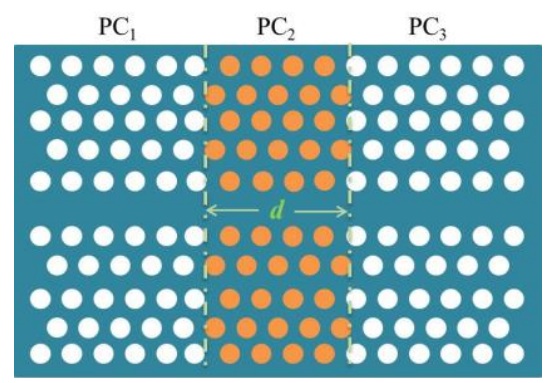

(a)

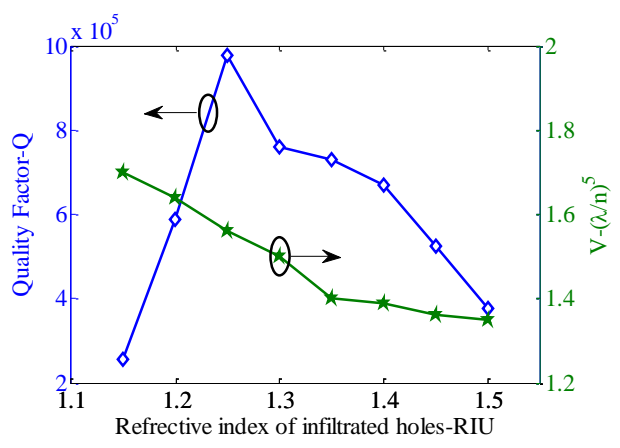

(b)

Fig. 15 Structure and resonant properties of heterostructure PCC formed by optofluidic infiltration ${ }^{[90]}$.

From the above studies, we can conclude that the reversibility of optofluidic infiltration combined with the degree of freedom in the fluid choice owned the following three advantages: First, the optofluidic PCCs are realized during the infiltration step, which can relax the constraint on the fabrication precision as compared to previous structure-based schemes for property optimization of PCCs. Second, these cavities can be configured in a flexible manner by altering the size or position of the infiltrated region as well as the RI of the liquid, offering a flexible, efficient and versatile method for the post-engineering and reconfiguring of PCCs. Third, these cavities exhibit high $Q$ factors despite the presence of a fluid. The versatility and flexibility of the optofluidic technology present great potential for the controls of PCC based devices, and the results offer perspectives for incorporating PCC devices in sensing circuits. If the structure is filled with liquid crystal, electro-optic or nonlinear polymer, there is also the possibility of tuning these structures when external voltage is applied.

\subsection{Cascaded PCC}

As discussed above, PCC sensors with high $Q$ factor and small volume can enhance the interaction between the analyte and incident light and improve the sensitivity to bulk properties. However, most of these sensors typically operate as point or single sensor and the number of targets which can be sensed at one time is relatively small. To overcome these drawbacks and realize multiple sensing sites, many sensor arrays based on cascaded PCCs have been developed 
[33, 70, 108-112]. In 2011, S. Pal et al. [70] proposed a multiplexed lgG sensor, in which three nanocavity coupled waveguides were placed in series. But the sensor volume was too large and not suitable for sensing application. While in 2012, Y. Wang et al. [108] developed a theoretical model of the integrated parallel self-collimation sensor array. But only three sensors could be integrated on the monolithic platform, which results in a low integration density. At the same time, D. Yang et al. [109] theoretically investigated the performances of nanoscale PC integrated RI sensor array on monolithic substrate by using some H0 cavities side-coupled resonant cavities (see Fig. 16(a)). The output resonant spectra of different cavities were independent with each other. In 2013 [85], this structure was also well used for multiple pressure sensors. Besides, S. Olyaee et al. [110] have also demonstrated that the sensor array could also be realized when some H1 cavities were side-coupled to a PCW. However, the main drawbacks of these side-coupled resonant cavity arrays were that the larger the number of PCCs integrated on the monolithic platform, the narrower the spacing of the resonant peaks of adjacent cavities was. Therefore, the sensing signal of each cavity might interact with each other due to the crosstalk in multi-cavity parallel sensors. And if the variation of one output signal caused by the target parameter change was too large, the shift of resonant wavelength might be greater than the spacing of adjacent resonant cavities. This will result in difficulties in recognizing the sensing signals from different cavities.

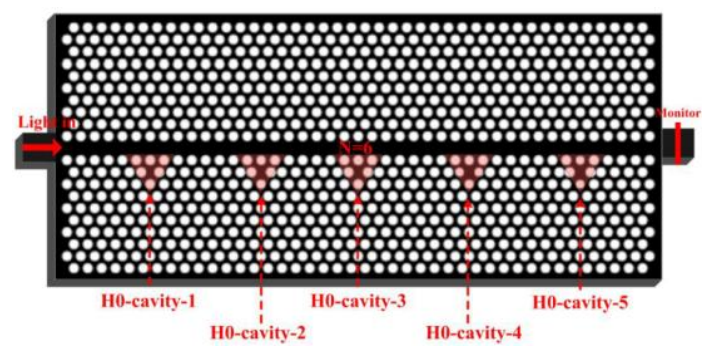

(a)

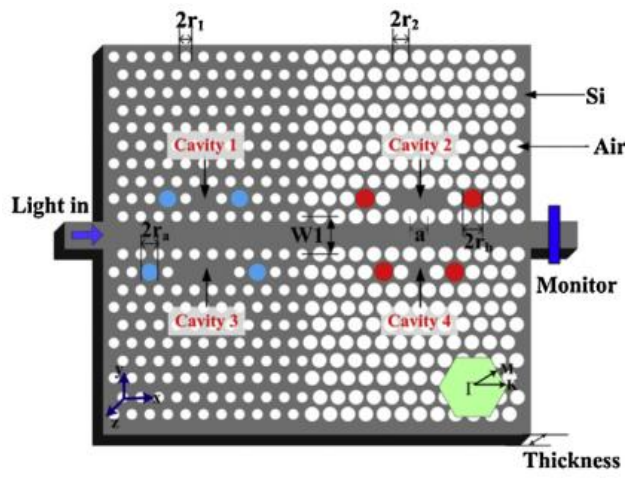

(b)

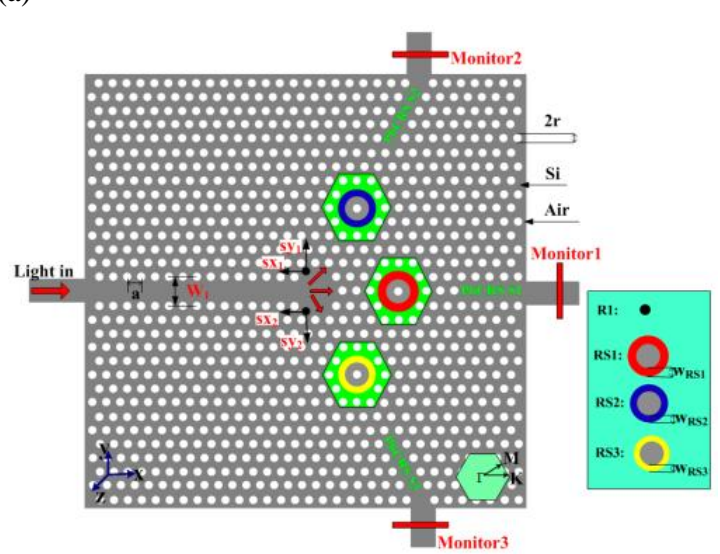

(c)

Fig. 16 Structures of cascaded PCC that published in Ref. [98], Ref. [100], and Ref. [101].

To efficiently enhance the integration density of sensor array and restrain crosstalk of adjacent PCCs, D. Yang et al. [33] further proposed a PC parallel resonant cavities design in 2014. The device was composed of some $\mathrm{H} 0$ cavities side-coupled to parallel output waveguides of an optimized PC beam-splitter. At last, the extinction ratio of the well-defined single notch exceeded $30 \mathrm{~dB}$, which allowed the implementation of simple but functional PC integrated sensor array, and eventually of more complex sensor networks. At the same time, Q. Liu et al. [111] proposed a radius-graded PC sensor array, where two L3 cavities and two $\mathrm{H} 1$ cavities were multiplexed and 
interlaced on both sides of a W1 PCW on the radius-graded PC slab (see Fig. 16(b)). And L. Huang et al. [112] demonstrated a low crosstalk ring-slot array structure used for label-free multiplexed sensing. The proposed sensor array was based on an array of three ring-slot and input/output line defect coupling waveguides (see Fig. 16(c)). Each ring-slot cavity had slightly different cavity spacing and different resonant frequency. Above all, the cascaded PCC can further improve the compactness and integration of PCC based sensor.

\subsection{Slow light assisted PCC}

Recently, slow light with a remarkably low group velocity has recently attracted wide attention, as it is regarded as a promising approach for time-domain processing of optical signal and spatial compression of optical energy [113]. In practical applications, as the light-matter interactions rely on the strength of the interaction between the optical field and the material, many nonlinear phenomena will be enhanced under the presence of slow light, which allows us to design miniaturized and high-sensitive devices based on this field enhancement [114-116]. In 2012, F. Hosseinibalam et al. [117] proposed a slow light assisted PCC for ultracompact, low power, and high-sensitive biosensor. The proposed biosensor was composed of a half-ring cavity that is side-coupled to an optofluidic slow light PCW (see Fig. 17(a)). Simulation results in Fig. 17(b) demonstrated that the sensitivity to RI changes could be increased from $77 \mathrm{~nm} / \mathrm{RIU}$ to 293 $\mathrm{nm} / \mathrm{RIU}$ when slow light was introduced. Then in 2013, W. C. Lai et al. [118] demonstrated that in photonic crystal sensors with a side-coupled cavity waveguide configuration, group velocity of the propagating mode in the coupled waveguide at the frequency of the resonant mode played an important role in enhancing the sensitivity. In linear L13 PCCs, with nearly same $Q$ factor of 7000 , the sensitivity could be increased from $57 \mathrm{~nm} / \mathrm{RIU}$ to $66 \mathrm{~nm} / \mathrm{RIU}$ when the group index of the coupled waveguide increased from 10.2 to 13.2. Therefore, it was concluded that in side-coupled PC cavity-waveguide sensors, in addition to the $Q$ of the uncoupled PCC and the optical mode overlap with analyte, slow light in the coupled PCW also contributed to the enhanced sensitivities of resonance modes. This is a promising method for enhancing the sensitivities of PCC based sensors.

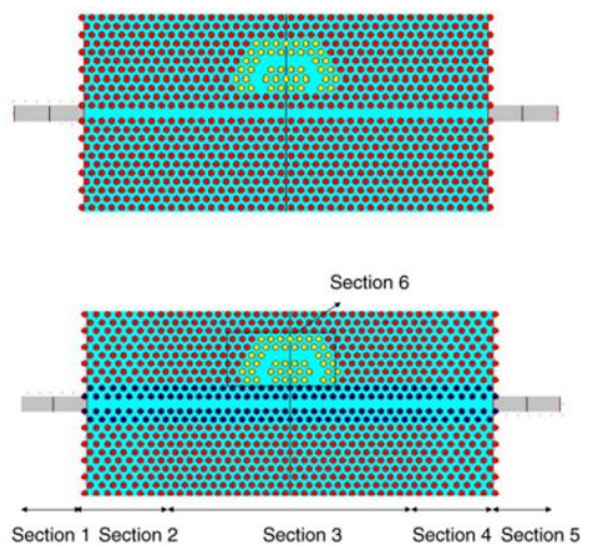

(a)

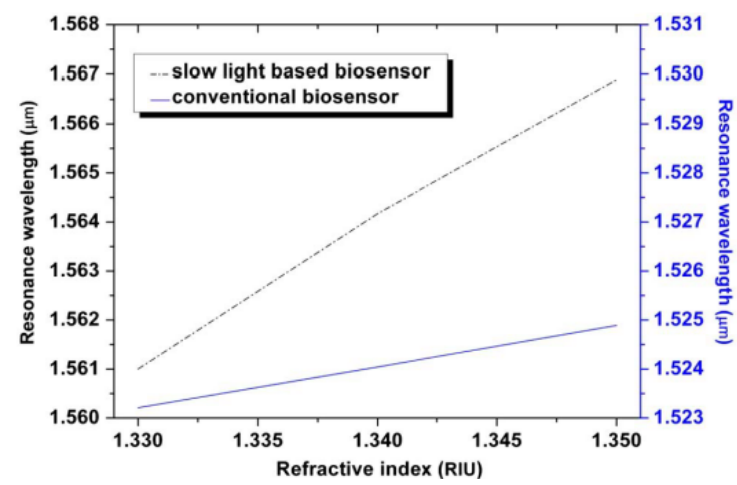

(b)

Fig. 17 Structures and the corresponding RI sensing property of ring PCC when slow light is introduced (by infiltrating optofluidic in the first two rows of air holes) and not introduced ${ }^{[106]}$.

\subsection{Functional PCC with optical coating}

In recent years, the deposition of optical coating with nanometric thickness has been shown to significantly enhance the sensitivity and selectivity of a number of optical sensing systems to 
certain external parameters, such as refractive index [119], $\mathrm{pH}$ [120], gas concentration [121], temperature [24], biochemical molecule [27], and humidity [122]. We can predict that the application ranges of PCC sensor can be greatly extended by combining optical coating with PCC to realize the functional PCC [121]. Besides, the technology of optical coating can also improve the sensitivity and selectivity of optical sensors based on the functional PCC with optical coating.

\section{Conclusion}

The review for the reported works and their corresponding results demonstrated that the PCCs have played very important roles in the optical sensor fields and will produce a significant industrial value. For each sensor type, the sensing principle, structure of PCC, and the corresponding sensing properties were described in detail. Besides, the new directions of PCCs for sensing applications were all discussed. From which, the readers who are interested in this field could not only see the unique properties and flexibilities in structural design of PCCs, but also broaden their thoughts and burst out some new solutions to further exploit the potentials of PCCs in ultra-compact and high-sensitive optical sensors. With the technology development of PC fabrication, much better design schemes of PCCs will be presented and much more PCC based optical sensors will be proposed. In the future, the key technologies of PCC based optical sensors will be the controllability, network, integration, all fiber, real-time measurements in fluidic environment, and the explorations on new mechanisms and new methods.

\section{Acknowledgments}

This work was supported in part by the National Science Foundation for Distinguished Young Scholars of China under Grant 61425003, the National Natural Science Foundation of China under Grant 61273059, the Fundamental Research Fund for the Central Universities under Grant N130604006 and N140404021, and State Key Laboratory of Synthetical Automation for Process Industries under Grant 2013ZCX09. 


\section{References:}

[1] E. Yablonovitch. Inhibited spontaneous emission in solid-state physics and electronics. Physical Review Letters, 1987, 58: 2059-2062.

[2] S. John. Strong localization of photons in certain disordered physics dielectric superlattices. Physical Review Letters, 1987, 58: 2486-2489.

[3] A. Scherer, O. Painter, J. Vuckovic, et al. Photonic crystals for confining, guiding, and emitting light. IEEE Transactions on Nanotechnology, 2002, 1(1): 4-11.

[4] J. D. Joannopoulos, P. R. Villeneuve, S. Fan. Photonic crystals: putting a new twist on light. Nature, 1997, 386(6621): 143-149.

[5] J. D. Joannopoulos, S. G. Johnson, J. N. Winn, et al. Photonic crystals: molding the flow of light. 2nd ed., Princeton, 2008.

[6] M. Notomi. Strong light confinement with periodicity. Proceedings of the IEEE, 2011, 99(10): 1768-1779.

[7] M. Arjmand, R. Talebzadeh. Optical filter based on photonic crystal resonant cavity. Optoelectronics and Advanced Materials-Rapid Communications, 2015, 9(1-2): 32-35.

[8] X. Ge, Y. Shi, S. He. Ultra-compact channel drop filter based on photonic crystal nanobeam cavities utilizing a resonant tunneling effect. Optics Letters, 2014, 39(24): 6973-6976.

[9] J. M. Brosi, C. Koos, L. C. Andreani, et al. High-speed low-voltage electro-optic modulator with a polymer-infiltrated silicon photonic crystal waveguide. Optics Express, 2008, 16(6): 4177-4191.

[10] Y. Gao, R. J. Shiue, X. Gan, et al. High-speed electro-optic modulator integrated with graphene-boron nitride heterostructure and photonic crystal nanocavity. Nano Letters, 2015, 15(3): 2001-2005.

[11] K. Fasihi. High-contrast all-optical controllable switching and routing in nonlinear photonic crystals. Journal of Lightwave Technology, 2014, 32(18): 3126-3131.

[12] K. Cui, Q. Zhao, X. Feng, et al. Thermo-optic switch based on transmission-dip shifting in a double-slot photonic crystal waveguide. Applied Physics Letters, 2012, 100(20): 201102(1-4).

[13] C. Y. Lin, H. Subbaraman, A. Hosseini, et al. Silicon nanomembrane based photonic crystal waveguide array for wavelength-tunable true-time-delay lines. Applied Physics Letters, 2012, 101(5): $\underline{051101(1-4) \text {. }}$

[14] Y. Zhao, Y. Zhang, Q. Wang. Research advances of photonic crystal gas and liquid sensors. Sensors and Actuators B-Chemical, 2011, 160(1): 1288-1297.

[15] R. V. Nair, R. Vijaya. Photonic crystal sensors: An overview. Progress in Quantum Electronics, 2010, 34(3): 89-134.

[16] C. Fenzl, T. Hirsch, O. S. Wolfbeis. Photonic crystals for chemical sensing and biosensing. Angewandte Chemie-International Edition, 2014, 53(13): 3318-3335.

[17] W. C. Lai, S. Chakravarty, X. Wang, et al. On-chip methane sensing by near-IR absorption signatures in a photonic crystal slot waveguide. Optics Letters, 2011, 36(6): 984-986.

[18] Y. Zhang, Y. Zhao, Q. Wang. Multi-component gas sensing based on slotted photonic crystal waveguide with liquid infiltration. Sensors and Actuators B-Chemical, 2013, 184: 179-188.

[19] Y. Zhang, Y. Zhao, Q. Wang. Measurement of methane concentration with cryptophane E infiltrated photonic crystal microcavity. Sensors and Actuators B-Chemical, 2015, 209: 431-437. 
[20] W. C. Lai, S. Chakravarty, Y. Zou, et al. Multiplexed detection of xylene and trichloroethylene in water by photonic crystal absorption spectroscopy. Optics Letters, 2013, 38(19): 3799-3802.

[21] Y. Chang, Y. Jhu, C. Wu. Temperature dependence of defect mode in a defective photonic crystal. Optics Communications, 2012, 285(6): 1501-1504.

[22] T. Lu, P. T. Lee. Ultra-high sensitivity optical stress sensor based on double-layered photonic crystal microcavity. Optics Express, 2009, 17(3): 1518-1526.

[23] Y. Liu, H. W. M. Salemink. All-optical on-chip sensor for high refractive index sensing in photonic crystals. EPL, 2014, 107(3): 34008(1-5).

[24] S. Zheng, B. Shan, M. Ghandehari, et al. Sensitivity characterization of cladding modes in long-period gratings photonic crystal fiber for structural health monitoring. Measurement, 2015, 72: 43-51.

[25] A. Casas-Bedoya, S. Shahnia, D. Di Battista, et al. Chip scale humidity sensing based on a microfluidic infiltrated photonic crystal. Applied Physics Letters, 2013, 103(18): $181109(1-4)$.

[26] S. Zheng, Y. Zhu, S. Krishnaswamy. Fiber humidity sensors with high sensitivity and selectivity based on interior nanofilm-coated photonic crystal fiber long-period gratings. Sensors and Actuators B-Chemical, 2013, 176: 264-274

[27] S. Zheng, Y. Zhu, S. Krishnaswamy. Nanofilm-coated photonic crystal fiber long-period gratings with modal transition for high chemical sensitivity and selectivity. In: Conference on Smart Sensor Phenomena, Technology, Networks, and Systems Integration, Mar. 12, 2012-Mar. 14, 2012, SPIE, San Diego, CA, 2012, 8346: 83460D.

[28] P. Lalanne, C. Sauwan, J. P. Hugonin. Photon confinement in photonic crystal nanocavities. Laser \& Photonics Reviews. 2008, 2(6): 514-526.

[29] S. Chakravarty, A. Hosseini, X. C. Xu, et al. Analysis of ultra-high sensitivity configuration in chip-integrated photonic crystal microcavity bio-sensors. Applied Physics Letters, 2014, 104(19): 191109(1-5).

[30] Y. Akahane, T. Asano, B. S. Song, et al. High-Q photonic nanocavity in a two-dimensional photonic crystal. Nature, 2003, 425(6961): 944-947.

[31] Y. Zhang, D. Li, C. Zeng, et al. Silicon optical diode based on cascaded photonic crystal cavities. Optics Letters, 2014, 39(6): 1370-1373.

[32] A. Majumdar, J. Kim, J. Vuckovic, et al. Electrical control of silicon photonic crystal cavity by graphene. Nano Letters, 2013, 13(2): 515-518.

[33] D. Yang, H. Tian, Y. Ji. Nanoscale low crosstalk photonic crystal integrated sensor array. IEEE Photonics Journal, 2014, 6(1): 4200107(1-7).

[34] Y. Fu, Y. Lee, S. Lin. Design and demonstration of high quality-factor H1-cavity in two-dimensional photonic crystal. Optics Letters, 2013, 38(22): 4915-4918.

[35] C. Caër, X. Le Roux, E. Cassan. High-Q silicon-on-insulator slot photonic crystal cavity infiltrated by a liquid. Applied Physics Letters, 2013, 103(25): 251106(1-4).

[36] B. Li, C. Lee. NEMS diaphragm sensors integrated with triple-nano-ring resonator. Sensors and Actuators A-Physical, 2011, 172(1): 61-68.

[37] D. Wang, Z. Yu, Y, Liu, et al. Ultrasmall modal volume and high Q factor optimization of a photonic crystal slab cavity. Journal of Optics, 2013, 15(12): 125102(1-7). 
[38] Y. Yang, D. Yang, H. Tian, et al. Photonic crystal stress sensor with high sensitivity in double directions based on shoulder-coupled aslant nanocavity. Sensors and Actuators A-Physical, 2013, 193: 149-154.

[39] Y. Zhang, Y. Zhao, D. Wu, et al. Fiber loop ring-down refractive index sensor based on high-Q photonic crystal cavity. IEEE Sensors Journal, 2014, 14(6): 1878-1885.

[40] A. F. Oskooi, D. Roundy, M. Ibanescu, et al. Meep: A flexible free-software package for electromagnetic simulations by the FDTD method. Computer Physics Communications, 2010, 181(3): 687-702.

[41] D. Dorfner, T. Zabel, T. Hürlimann, et al. Photonic crystal nanostructures for optical biosensing applications. Biosensors \& Bioelectronics, 2009, 24(18): 3688-3692.

[42] I. M. White, X. Fan. On the performance quantification of resonant refractive index sensors. Optics Express, 2008, 16(2): 1020-1028.

[43] E. Chow, A. Grot, L. W. Mirkarimi, et al. Ultracompact biochemical sensor built with two-dimensional photonic crystal microcavity. Optics Letters, 2004, 29(10): 1093-1095.

[44] X. Wang, Z. Xu, N. Lu, et al. Ultracompact refractive index sensor based on microcavity in the sandwiched photonic crystal waveguide structure. Optics Communications, 2008, 281(6): 1725-1731.

[45] D. F. Dorfner, T. Hürlimann, T. Zabel, et al. Silicon photonic crystal nanostructures for refractive index sensing. Applied Physics Letters, 2008, 93(18): 181103(1-3).

[46] L. A. Shiramin, R. Kheradmand, A. Abbasi. High-sensitive double-hole defect refractive index sensor based on 2-D photonic crystal. IEEE Sensors Journal, 2013, 13(5): 1483-1486.

[47] J. Zhou, H. Tian, D. Yang, et al. Integration of high transmittance photonic crystal H2 nanocavity and broadband W1 waveguide for biosensing applications based on silicon-on-insulator substrate. Optics Communications, 2014, 330: 175-183.

[48] L. Huang, H. Tian, D. Yang, et al. Optimization of figure of merit in label-free biochemical sensors by designing a ring defect coupled resonator. Optics Communications, 2014, 332: $42-49$.

[49] L. Huang, H. Tian, J. Zhou, et al. Label-free optical sensor by designing a high-Q photonic crystal ring-slot structure. Optics Communications, 2015, 335: 73-77.

[50] A. Di Falco, L. O’Faolain, T. F. Krauss. Photonic crystal slotted slab waveguides. Photonics and Nnostructures-Fundamentals and Applications, 2008, 6(1): 38-41.

[51] C. Caer, X. Le Roux, E. Cassan. Enhanced localization of light in slow wave slot photonic crystal waveguides. Optics Letters, 2012, 37(17): 3660-3662.

[52] V. Almeida, Q. Xu, C. A. Barrios, et al. Guiding and confining light in void nanostructure. Optics Letters, 2004, 29(11): 1209-1211.

[53] T. Yamamoto, M. Notomi, H. Taniyama, et al. Design of a high-Q air-slot cavity based on a width-modulated line-defect in a photonic crystal slab. Optics Express, 2008, 16(18): 13809-13817.

[54] A. Di Falco, L. O'Faolain, T. F. Krauss. Chemical sensing in slotted photonic crystal heterostructure cavities. Applied Physics Letters, 2009, 94(6): 063503(1-3).

[55] S. H. Mirsadeghi, E. Schelew, J. F. Young. Photonic crystal slot-microcavity circuit implemented in silicon-on-insulator: High Q operation in solvent without undercutting. Applied Physics Letters, 2013, 102(13): 131115(1-4). 
[56] C. Caër, S. F. Serna-Otálvaro, W. Zhang, et al. Liquid sensor based on high-Q slot photonic crystal cavity in silicon-on-insulator configuration. Optics Letters, 2014, 39(20): 5792-5794.

[57] P. Bing, J. Yao, Y. Lu, et al. A surface-plasmon-resonance sensor based on photonic-crystal-fiber with large size microfluidic channels. Optica Applicata, 2012, 42(3): 493-501.

[58] B. Li, L. Jiang, S. Wang, et al. A new Mach-Zehnder interferometer in a thinned-cladding fiber fabricated by electric arc for high sensitivity refractive index sensing. Optics and Lasers in Engineering, 2012, 50(6): 829-832.

[59] G. Quero, A. Crescitelli, D. Paladino, et al. Evanescent wave long-period fiber grating within D-shaped optical fibers for high sensitivity refractive index detection. Sensors and Actuators B-Chemical, 2011, 152(2): 196-205.

[60] R. Gao, Y. Jiang, W. Ding, et al. Filmed extrinsic Fabry-Perot interferometric sensors for the measurement of arbitrary refractive index of liquid. Sensors and Actuators B-Chemical, 2013, 177: 924-928.

[61] S. Chakravarty, J. Topol'ančik, P. Bhattacharya, et al. Ion detection with photonic crystal microcavities. Optics Letters, 2005, 30(19): 2578-2580.

[62] W. C. Lai, S. Chakravarty, Y. Zou, et al. Silicon nano-membrane based photonic crystal microcavities for high sensitivity bio-sensing. Optics Letters, 2012, 37(7): 1208-1210.

[63] Y. Zou, S. Chakravarty, D. N. Kwong, et al. Cavity-waveguide coupling engineered high sensitivity silicon photonic crystal microcavity biosensors with high yield. IEEE Journal of Selected Topics in Quantum Electronics, 2014, 20(4): 6900710(1-10).

[64] M. R. Lee, P. M. Fauchet. Two-dimensional silicon photonic crystal based biosensing platform for protein detection. Optics Express, 2007, 15(8): 4530-4535.

[65] M. R. Lee, P. M. Fauchet. Nanoscale microcavity sensor for single particle detection. Optics Letters, 2007, 32(22): 3284-3286.

[66] S. Zlatanovic, L. W. Mirkarimi, M. M. Sigalas, et al. Photonic crystal microcavity sensor for ultracompact monitoring of reaction kinetics and protein concentration. Sensors and Actuators B-Chemical, 2009, 141(1): 13-19.

[67] F. L. Hsiao, C. Lee. Computational study of photonic crystals nano-ring resonator for biochemical sensing. IEEE Sensors Journal, 2010, 10(7): 1185-1191.

[68] F. L. Hsiao, C. Lee. Nanophotonic biosensors using hexagonal nanoring resonators: computational study. Journal of Micro-Nanolithography MEMS and MOEMS, 2011, 10(1): $\underline{013001(1-8)}$.

[69] C. P. Ho, B. Li, A. J. Danner, et al. Design and modeling of 2-D photonic crystals based hexagonal triple-nano-ring resonators as biosensors. Microsystem Technologies-Micro-and Nanosystems-Information Storage and Processing Systems, 2013, 19(1): 53-60.

[70] S. Pal, E. Guillermain, R. Sriram, et al. Silicon photonic crystal nanocavity-coupled waveguides for error-corrected optical biosensing. Biosensors and Bioelectronics, 2011, 26(10): 4024-4031.

[71] S. Pal, A. R. Yadav, M. A. Lifson, et al. Selective virus detection in complex sample matrices with photonic crystal optical cavities. Biosensors and Bioelectronics, 2013, 44: 229-234.

[72] M. G. Scullion, A. Di Falco, T. F. Krauss. Slotted photonic crystal cavities with integrated microfluidics for biosensing applications. Biosensors and Bioelectronics, 2011, 27(1): 101-105. 
[73] T. Sünner, T. Stichel, S. -H. Kwon, et al. Photonic crystal cavity based gas sensor. Applied Physics Letters, 2008, 92(26): 261112(1-3).

[74] J. Jágerská, H. Zhang, Z. Diao, et al. Refractive index sensing with an air-slot photonic crystal nanocavity. Optics Letters, 2010, 35(15): 2523-2525.

[75] K. Li, J. Li, Y. Song, et al. $\mathrm{L}_{n}$ slot photonic crystal microcavity for refractive index gas sensing. IEEE Photonics Journal, 2014, 6(5): 6802509(1-9).

[76] T. Stomeo, M. Grande, A. Qualtieri, et al. Fabrication of force sensors based on two-dimensional photonic crystal technology. Microelectronic Engineering, 2007, 84(5-8): 1450-1453.

[77] C. Lee, R. Radhakrishnan, C. Chen, et al. Design and modeling of a nanomechanical sensor using silicon photonic crystals. Journal of Lightwave Technology, 2008, 26(5-8): 839-846.

[78] C. Lee, J. Thillaigovindan. Optical nanomechanical sensor using a silicon photonic crystal cantilever embedded with a nanocavity resonator. Applied Optics, 2009, 48(10): 1797-1803.

[79] C. Lee, J. Thillaigovindan, C. C. Chen, et al. Si nanophotonics based cantilever sensor. Applied Physics Letters, 2008, 93(11): 113113(1-3).

[80] T. T. Mai, F. L. Hsiao, C. Lee, et al. Optimization and comparison of photonic crystal resonators for silicon microcantilever sensors. Sensors and Actuators A-Physical, 2011, 165(1): 16-25.

[81] B. Li, F. L. Hsiao, C. Lee. Configuration analysis of sensing element for photonic crystal based NEMS cantilever using dual nano-ring resonator. Sensors and Actuators A-Physical, 2011, 169(2): 352-361.

[82] B. T. Tung, D. V. Dao, T. Ikeda, et al. Investigation of strain sensing effect in modified single-defect photonic crystal nanocavity. Optics Express, 2011, 19(9): $\underline{821(1-9)}$.

[83] D. Yang, H. Tian, Y. Ji. Microdisplacement sensor based on high-Q nanocavity in slot photonic crystal. Optical Engineering, 2011, 50(5): 054402(1-6).

[84] D. Mao, P. Liu, K. M. Ho, et al. A theoretical study of a nano-opto-mechanical sensor using a photonic crystal-cantilever cavity. Journal of Optics, 2012, 14(7): 075002(1-8).

[85] D. Yang, H. Tian, N. Wu, et al. Nanoscale torsion-free photonic crystal pressure sensor with ultra-high sensitivity based on side-coupled piston-type microcavity. Sensors and Actuators A-Physical, 2013, 199: 30-36.

[86] Y. Yang, H. Tian, D. Yang, et al. Nanomechanical three dimensional force photonic crystal sensor using shoulder-coupled resonant cavity with an inserted pillar. Sensors and Actuators A-Physical, 2014, 209: 33-40.

[87] D. Yang, H. Tian, Y. Ji. The properties of lattice-shifted microcavity in photonic crystal slab and its applications for electro-optical sensor. Sensors and Actuators A-Physical. 2011, 171(2): 146-151.

[88] Y. Zhao, Y. Zhang, R. Lv. Simultaneous measurement of magnetic field and temperature based on magnetic fluid-infiltrated photonic crystal cavity. Sensors and Actuators B-Chemical. 2015, 64(4): 1055-1062.

[89] L. O'Faolain, T. P. White, D. O'Brien, et al. Dependence of extrinsic loss on group velocity in photonic crystal waveguides. Optics Express, 2007, 15(20): 13129-13138.

[90] D. M. Beggs, L. O'Faolain, T. F. Krauss. Accurate determination of the functional hole size in photonic crystal slabs using optical methods. Photonics and Nanostructures Fundamentals and Applications, 2008, 6(3-4): 213-218. 
[91] D. Pergande, T. M. Geppert, A. von Rhein, et al. Miniature infrared gas sensors using photonic crystals. Journal of Applied Physics, 2011, 109(8): 083117(1-7).

[92] H. Hagina, Y. Takahashi, Y. Tanaka, et al. Effects of fluctuation in air hole radii and positions on optical characteristics in photonic crystal heterostructure nanocavities. Physical Review B. 2009, 79(8): 085112(1-8).

[93] C. Karnutsch, C. L. C. Smith, A. Graham, et al. Temperature stabilization of optofluidic photonic crystal cavities. Applied Physics Letters, 2009, 94(23): 231114(1-3).

[94] T. Asano, B. S. Song, S. Noda. Analysis of the experimental Q factors ( 1 million) of photonic crystal nanocavities. Optics Express, 2006, 14(5): 1996-2002.

[95] C. Monat, P. Domachuk, B. J. Eggleton. Integrated optofluidics: A new river of light. Nature Photonics, 2007, 1(2): 106-114.

[96] F. Intonti, S. Vignolini, V. Türck, et al. Rewritable photonic circuits. Applied Physics Letters, 2006, 89(21): 211117(1-3).

[97] M. Ebnali-Heidari, F. Koohi-Kamali, A. Ebnali-Heidari, et al. Designing tunable microstructure spectroscopic gas sensor using optofluidic hollow-core photonic crystal fiber. IEEE Journal of Quantum Electronics, 2014, 50(12): 943-950.

[98] Y. Zhang, Y. Zhao, J. Li. Theoretical research on slow light engineering of slotted photonic crystal waveguides with elliptical holes and optofluidic infiltration. Applied Optics, 2015, 54(7): 1639-1646.

[99] M. Hosseinpour, M. Ebnali-Heidari, M. Kamali, et al. Optofluidic photonic crystal slow light coupler. Journal of the Optical Society of America B-Optical Physics, 2013, 30(3): 717-722.

[100] N. W. L. Speijcken, M. A. Dündar, A. C. Bedoya, et al. In situ optofluidic control of reconfigurable photonic crystal cavities. Applied Physics Letters, 2012, 100(26): $\underline{261107(1-5)}$.

[101] H. H. J. E. Kicken, P. F. A. Alkemade, R. W. van der Heijden, et al. Wavelength tuning of planar photonic crystals by local processing of individual holes. Optics Express, 2009, 17(24): 22005-22011.

[102] C. L. C. Smith, D. K. C. Wu, M. W. Lee, et al. Microfluidic photonic crystal double heterostructures. Applied Physics Letters, 2007, 91(12): 121103(1-3).

[103] S. Tomljenovic-Hanic, C. M. de Sterke, M. J. Steel. Design of high-Q cavities in photonic crystal slab heterostructures by air-holes infiltration. Optics Express, 2006, 14(25): 12451-12456.

[104] C. L. C. Smith, U. Bog, S. Tomljenovic-Hanic, et al. Reconfigurable microfluidic photonic crystal slab cavities. Optics Express, 2008, 16(20): 15887-15896.

[105] U. Bog, C. L. C. Smith, M. W. Lee, et al. High-Q microfluidic cavities in silicon-based two-dimensional photonic crystal structures. Optics Express, 2008, 33(19): 2206-2208.

[106] A. C. Bedoya, S. Mahmoodian, C. Monat, et al. Liquid crystal dynamics in a photonic crystal cavity created by selective microfluidic infiltration. Optics Express, 2010, 18(26): 27280-27290.

[107] F. Intonti, S. Vignolini, F. Riboli, et al. Tuning of photonic crystal cavities by controlled removal of locally infiltrated water. Applied Physics Letters, 2009, 95(17): 173112(1-3).

[108] Y. Wang, H. Wang, Q. Xue, et al. Photonic crystal self-collimation sensor. Optics Express, 2012, 20(11): 12111-12118. 
[109] D. Yang, H. Tian, Y. Ji. Nanoscale photonic crystal sensor arrays on monolithic substrates using side-coupled resonant cavity arrays. Optics Express, 2011, 19(21): 20023-20034.

[110] S. Olyaee, S. Najafgholinezhad. Computational study of a label-free biosensor based on a photonic crystal nanocavity resonator. Applied Optics, 2013, 52(29): 7206-7213.

[111] Q. Liu, H. Tian, D. Yang, et al. Nanoscale radius-graded photonic crystal sensor arrays using interlaced and symmetrical resonant cavities for biosensing. Sensors and Actuators A-Physical, 2014, 216: 223-230.

[112] L. Huang, H. Tian, J. Zhou, et al. Design low crosstalk ring-slot array structure for label-free multiplexed sensing. Sensors, 2014, 14(9): 15658-15668.

[113] J. B. Khurgin, R. S. Tucker. Slow light: Science and applications. CRC Press, Boca Raton, 2008.

[114] S. Bakhshi, M. K. Morawej-Farshi, M. Ebnali. Design of an ultracompact low-power all-optical modulator by means of dispersion engineered slow light regime in a photonic crystal Mach-Zehnder interferometer. Applied Optics, 2012, 51(14): 2687-2692.

[115] Y. Zhang, H. Tian, X. Zhang, et al. Experimental evidence of enhanced rotation sensing in a slow-light structure. Optics Letters, 2010, 35(5): 691-693.

[116] Y. Zhang, Y. Zhao, Q. Wang. Improved design of slow light interferometer and its application in FBG displacement sensor. Sensors and Actuators A-Physical, 2014, 214: 168-174.

[117] F. Hosseinibalam, S. Hassanzadeh, A. Ebnali-Heidari, et al. Design of an optofluidic biosensor using the slow-light effect in photonic crystal structures. Applied Optics, 2012, 51(5): 568-576.

[118] W. C. Lai, S. Chakravarty, Y. Zou, et al. Slow light enhanced sensitivity of resonance modes in photonic crystal biosensors. Applied Physics Letters, 2013, 102(4): 041111(1-4).

[119] M. Smietana, D. Brabant, W. J. Bock, et al. Refractive-index sensing with inline core-cladding intermodal interferometer based on silicon nitride nano-coated photonic crystal fiber. Journal of Lightwave Technology, 2012, 30(8): 1185-1189.

[120] P. Hu, X. Dong, W. C. Wong, et al. Photonic crystal fiber interferometric pH sensor based on polyvinyl alcohol/polyacrylic acid hydrogel coating. Applied Optics, 2015, 54(10): 2647-2652.

[121] Y. Wang, D. N. Wang, F. Yang, et al. Sensitive hydrogen sensor based on selectively infiltrated photonic crystal fiber with Pt-loaded $\mathrm{WO}_{3}$ coating. Optics Letters, 2014, 39(13): 3872-3875.

[122] S. Zheng. Long-period fiber grating moisture sensor with nano-structured coatings for structural health monitoring. Structural Health Monitoring-An International Journal, 2015, 14(2): 148-157. 


\section{Biography:}

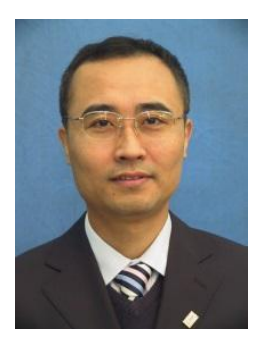

Yong Zhao received his M.A. and Ph.D. degrees, respectively, in precision instrument \& automatic measurement with laser and fiber-optic techniques from the Harbin Institute of Technology, China, in 1998 and 2001. He was awarded a first prize scholarship in 2000 by the China Instrument and Control Society and the Sintered Metal Corporation (SMC) scholarship in Japan. He was a scholarship in Japan. He was a postdoctor in the Department of Electronic Engineering of Tsinghua University from 2001 to 2003, and then worked as an associate professor in the Department of Automation, Tsinghua University of China. In 2006, he was a visiting scholar of University of Illinois in Urbana and Champagne, USA. In 2008, he was awarded as the "New Century Excellent Talents in University" by the Ministry of Education of China. In 2009, he was awarded as the "Liaoning Bai-Qian-Wan Talents" by Liaoning Province. In 2011, he was awarded by the Royal Academy of Engineering as an academic researcher of City University London. He was awarded by the National Science Foundation for Distinguished Young Scholars of China, in 2014. Now he is working in Northeastern University as a full professor. As a leader of his research group, his current research interests are the development of fiber-optic sensors and device, fiber Bragg grating sensors, novel sensor materials and principles, slow light and sensor technology, optical measurement technologies. He has authored and co-authored more than 180 scientific papers and conference presentations, 7 patents, and 4 books. He is a member in the Editorial Boards of the international journals of Sensor Letters, Instrumentation Science \& Technology, Journal of Sensor Technology, and Advances in Optical Technologies.

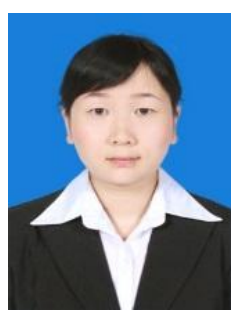

Ya-nan Zhang was born in Anhui, China, in June 1989. She received her B.A. and M.A. degrees, respectively, in 2010 and 2012 from the College of Information Science and Engineering, Northeastern University, Shenyang, China, where she is currently working toward the Ph.D. degree. Her research interests include fiber optical sensors, photonic crystal waveguide sensors, and slow light technology and its sensing applications. She has authored and co-authored more than 25 scientific papers and conference presentations.

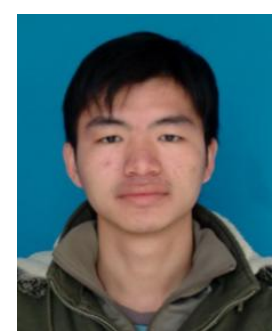

Ri-qing Lv was born in Guangdong, China, in 1985. He received his B.S, M.A. and $\mathrm{Ph} . \mathrm{D}$. degrees, respectively, in biomedical engineering, circuit and system, detection technology and automatic equipment from Northeastern University, Shenyang, China, in 2008, 2010 and 2014. He is currently working in the College of Information Science and Engineering at Northeastern University as a postdoctor. His research interests are magnetic fluid and fiber-optic sensors. He has authored and co-authored about 20 scientific papers, patents and conference presentations. 


\section{List of the figure captions}

Fig. 1 Schematic structures of (a) L4 PCC, (b) H0 PCC, (c) mode-gap PCC, (d) ring PCC, and (e) shoulder-coupled PCC.

Fig. 2 Schematic structure of PCW (a) and its corresponding electric field distribution (b) ${ }^{[36]}$.

Fig. 3 (a) Electric field distribution of a shoulder-coupled PCC, and (b) transmission spectra of W1 PCW and shoulder-coupled PCC ${ }^{[36]}$.

Fig. 4 Transmission spectra of PCC when the RI of defected holes is changed from 1.330 to 1.350 with an interval of $0.005^{[36]}$.

Fig. 5 Schematic structures of (a) ring PCC ${ }^{[60]}$ and (b) cascaded ring PCC ${ }^{[61]}$ that used for biochemical sensors, and SEM images of (c) H0 cavity ${ }^{[63]}$ and (d) slot PCC ${ }^{[65]}$ that used for biochemical sensors.

Fig. 6 Structure of heterostructure PCC (a), the corresponding transmission spectra for vacuum, nitrogen, $\mathrm{SF}_{6}$ gases (b), and the relationship between resonant wavelength shift and surrounding pressure (c) ${ }^{[66]}$.

Fig. 7 Structures of heterostructure slot PCC ${ }^{[67]}$ and $\mathrm{L} n$ slot PCCs ${ }^{[68]}$ for gas sensing.

Fig. 8 Schematic structure and the corresponding transmission spectra of cryptophane E infiltrated PCC ${ }^{[69]}$.

Fig. 9 Structures of shoulder-coupled PCC in (a) suspended silicon bridge ${ }^{[71]}$ and (b) silicon cantilever ${ }^{[72]}$ for mechanical sensing.

Fig. 10 Structures of one ring PCC ${ }^{[74]}$, dual ring PCC ${ }^{[33]}$, and triple ring PCC ${ }^{[75]}$ for mechanical sensing.

Fig. 11 (a) Structure of H0 slot cavity for displacement sensing ${ }^{[77]}$ and (b) structure of PCC integrated with cantilever for stress sensing ${ }^{[78]}$.

Fig. 12 Structures of shoulder-coupled PCC ${ }^{[35]}$, piston-typed PCC ${ }^{[79]}$, and modular 
$\mathrm{PCC}^{[80]}$ for force sensing.

Fig. 13 Schematic structure and the corresponding sensing property of polymer infiltrated PCC ${ }^{[87]}$.

Fig. 14 Schematic structure cascaded H0 PCC with magnetic field infiltration ${ }^{[88]}$.

Fig. 15 Structure and resonant properties of heterostructure PCC formed by optofluidic infiltration ${ }^{[90]}$.

Fig. 16 Structures of cascaded PCC that published in Ref. [98], Ref. [100], and Ref. [101].

Fig. 17 Structures and the corresponding RI sensing property of ring PCC when slow light is introduced (by infiltrating optofluidic in the first two rows of air holes) and not introduced ${ }^{[106]}$.

Table I Comparison of different PCCs that used for RI sensors and their sensing properties.

Table II Comparison of the presented optical systems that used for RI measurement 